PFC/JA-89-31

PULSE SHAPES FOR ABSOLUTE AND CONVECTIVE

CYCLOTRON-RESONANCE-MASER INSTABILITIES

John A. Davies*

- Ronald C. Davidson

George L. Johnston

July, 1989

Plasma Fusion Center
Massachusetts Institute of Technology
Cambridge, MA 02139

*Permanent address: Clark University, Worcester, MA 01610

Research supported in part by the Department of Energy High Energy Physics Division, the Naval Research Laboratory Plasma Physics Division, and the Office of Naval Research. 


\title{
PULSE SHAPES FOR ABSOLUTE AND CONVECTIVE CYCLOTRON-RESONANCE-MASER INSTABILITIES
}

\author{
John A. Davies \\ Clark University \\ Worcester, MA 01610 \\ and \\ Ronald C. Davidson and George L. Johnston \\ Plasma Fusion Center \\ Massachusetts Institute of Technology \\ Cambridge, MA 02193
}

\begin{abstract}
This paper contains a linear analysis of pulse shapes produced by a spatial and temporal delta-function disturbance of cyclotron-resonance-maser modes for the case where the initial equilibrium state is free of radiation. A pinch-point analysis based on the theory of Briggs and Bers is employed. Numerical and analytical techniques are developed for the straightforward calculation of pinch-point coordinates in a reference frame moving with arbitrary velocity in the axial direction. Examples analyzed include the absolute instability in the waveguide operating mode, in higher harmonics of the operating mode, and in lower-frequency waveguide modes when the operating mode is a higher-order waveguide mode. Effects of waveguide wall resistance on pulse shapes and the effectiveness of such resistance in suppressing or reducing the growth rates of absolute instabilities are also analyzed.
\end{abstract}




\section{INTRODUCTION}

During the past three decades considerable interest has developed in the employment of the cyclotron-resonance-maser instability for the efficient generation of high power radiation with centimeter to submillimeter wavelengths (Schneider 1959; Flyagin et al. 1977; Bratman et al. 1981; Fliflet 1986; Chu \& Lin 1988). Devices employing this instability are generally referred to as cyclotron resonance masers (CRMs). The two principal operating regimes for the CRM are that in which the phase velocity of waveguide radiation is much greater than $c$ and that in which the phase velocity is close to $c$. Devices operating in the former regime are referred to as gyrotrons, whereas those operating in the latter regime are referred to as cyclotron autoresonance masers (CARMs). Much of the work presented in this paper is an outgrowth of theoretical and computational work done in support of present and proposed CARM experiments at M.I.T. (Pendergast et al. 1988; Danly et al. 1988; Danly et al. 1989). This paper also compliments an earlier paper on pulse shapes in the free electron laser (FEL) (Davies, Davidson \& Johnston 1988).

A problem which must be addressed in CRM design is the presence of absolutely unstable CRM modes (Lau et al. $1981 a$, b; Lin, Chu \& Bromborsky 1987; Chu \& Lin 1988; Lin, Lin \& Chu 1988; Davies 1989). The growth of radiation in such modes may suppress the growth of radiation in the desired CRM mode, which is typically a convective mode. An instability in an unstable medium is convective if the response of the medium to a perturbation (localized in time and space) propagates as a growing pulse away from its source. The instability is absolute if the response is a growing pulse which encompasses its source. The nonrelativistic, linear theory of the shapes of such pulses is given by Briggs (1964) and Bers (1983). The relativistic theory appears in Bers, Ram \& Francis (1984), and a detailed summary of the latter work is given in Davies et al. (1988). Consequently, we give only a brief, qualitative summary of the theory. The response of a one-dimensional, spatially-unbounded medium to a delta-function disturbance in space and time can be expressed in the form of an inverse temporal Laplace transform and an inverse spatial Fourier transform. The laboratory-frame dispersion relation for a system $[D(k, w)=0]$ gives rise to images in the complex $k$-plane of contours in the complex $\omega$-plane. In particular, the 
contour for the inverse Laplace transform (referred to as the $L$-contour), has images in the complex $k$-plane. The existence of absolute instability is determined by lowering the $L$-contour towards the real $\omega$-axis and observing the behavior of its images in the complex $k$-plane. If during this process two $L$-contour images (one from above the real $k$-axis and one from below this axis) merge to form a saddle point in $\omega(k)$, then absolute instability is present. Such a saddle point is called a pinch point, and it is characterized by the complex coordinates $\left(k_{s}, \omega_{s}\right)$ of the saddle point. The temporal growth rate of the absolute instability is given by $\operatorname{Im}\left(\omega_{s}\right)>0$. Pulse shapes, giving the time-asymptotic response of the system to a delta-function disturbance $\delta(z) \delta(t)$ are obtained by carrying out such a pinch-point analysis for a general reference frame moving with an arbitrary normalized velocity $\beta_{v}=v / c$ in the spatial direction (z) of variation of the one-dimensional system. The general-frame dispersion relation is $D_{v}\left(k^{\prime}, \omega^{\prime}\right)=D\left(k\left(k^{\prime}, \omega^{\prime}\right), \omega\left(k^{\prime}, \omega^{\prime}\right)\right)=0$, where $(k, \omega)$ and $\left(k^{\prime}, \omega^{\prime}\right)$ are related by the Lorentz transformation. The time-asymptotic pulse amplitude $G(z, t)$ (relative to the laboratory frame) is given by $\ln G(z=v t, t) \sim t \operatorname{Im}\left(\omega_{s}^{\prime}\right) / \gamma_{v}$, where $\omega_{3}^{\prime}$ refers to the pinch point in the general frame and $\gamma_{v}=\left(1-\beta_{v}^{2}\right)^{-\frac{1}{2}}$. Such a pulse is conveniently represented by a plot of $t^{-1} \ln G(z=v t, t)=\operatorname{Im}\left(\omega_{s}^{\prime}\right) / \gamma_{v}$ vs. $\beta_{v}$. If the pulse encompasses the point $\beta_{v}=0$, absolute instability is present. The growth rate of the absolute instability is the height of the pulse at $\beta_{v}=0$.

The purposes of this paper are to investigate pulse shapes for the CRM and concurrently to present numerical and approximate analytic methods for determining absolute instability growth rates both for the case of zero and nonzero waveguide-wall resistance. The feasibility of suppressing absolute instability by introducing waveguide-wall resistance is also investigated. The treatment in this paper is limited to the case of $\mathrm{TE}_{m n}$ waveguide modes. In $\$ 2$ the laboratory-frame CRM dispersion relation, for the case of zero wall resistance, is extended to a general reference frame moving with arbitrary velocity $\beta_{v} c$ in the $z$-direction. The simplified form of this dispersion relation used in the analysis is given by (7). In $\S 3$ straightforward techniques are developed for determining pinch points of (7). Approximate expressions are determined for general-frame pinch-point coordinates valid when $s b^{\prime}>1$, where $s$ is the beam harmonic and $b^{\prime}$ is the general-frame normalized applied magnetic field strength in (8). These approximations appear in (25)-(27). We have not 
developed approximate expressions for pinch-point coordinates over intervals of $\beta_{v}$ where $s b^{\prime}<1$. However, at the end of $\S 3$, a simple, straightforward method is presented for numerically computing exact pinch-point coordinates when $s b^{\prime}<1$.

In $\S 4$ several numerically computed examples of CRM pulses are presented. For an applied axial magnetic field sufficiently stronger in comparison with the grazing field (typical of the CARM), there are two pulses: an upshifted pulse which is convective, and a downshifted pulse which may show absolute instability. In normal operating regimes, the FEL also has two such pulses. However, the two CRM pulses are connected at a value of $\beta_{v}$ approximately equal to the beam velocity, whereas typically FEL pulses are separated. As the applied magnetic field is reduced to (or below) the grazing value (typical of the gyrotron), the CRM pulses merge into a single pulse. Examples are presented for absolute instability in the waveguide operating mode, absolute instability in higher harmonics, and absolute instability due to operation in a waveguide mode above the lowest frequency waveguide mode.

In $\S 5$ we analyze finite skin-depth effects on pulse shapes and on growth rates of absolute instabilities. In $\S 5.1$ the laboratory-frame CRM dispersion relation with $\delta / r_{w}>$ 0 is extended to the general reference frame for the special case of $\mathrm{TE}_{0 n}$ modes. For simplicity, it is also assumed that the waveguide-wall permittivity and permeability are exactly one in the laboratory frame. In a numerical example, it is found that the effect of increasing $\delta / r_{w}$ is to move the left edge of the pulse to the right (i.e. to larger values of $\beta_{v}$ ). A shift of this edge from negative to positive $\beta_{v}$ converts an absolute instability into a convective instability. In $\$ 5.2$ we consider the effectiveness of a nonzero value of $\delta / r_{w}$ in suppressing or a least reducing the growth rate of an absolute instability. The treatment includes $\mathrm{TE}_{m n}$ modes for arbitrary values of $m$. The discussion covers the range of $0.5<\beta_{\|}<1.0$, which is the range of interest for the CARM. Figure 11 can be used to determine whether or not the use of wall resistance to suppress an absolutely unstable mode is a practical possibility. Approximate analytical formulas giving the effects of $\delta / r_{w}$ on laboratory-frame pinch-point coordinates are developed. Equations (48)-(50) are applicable to the case where $s b>1$. Equations (51) and (52) are of general applicability and give finite wall-resistance corrections to previously computed pinch-point coordinates 
for $\delta / r_{w}=0$. Two numerical examples are presented in $\S 5.2$. In the first example, the waveguide-wall resistance is found to substantially reduce the growth rate of an absolute instability in the second beam harmonic when the operating mode is the first harmonic for a $\mathrm{TE}_{11}$ mode. In the second example, wall resistance is found to be ineffective in reducing the growth rate of an absolute instability in the $\mathrm{TE}_{11}$ mode when the operating mode is the higher-order $\mathrm{TE}_{13}$ mode.

In the Appendix, the effects of wall resistance at very small small values of $\beta_{\|}$are discussed. In this regime, it is shown that wall resistance is destabilizing instead of stabilizing. 


\section{GENERAL-FRAME DISPERSION RELATION}

We consider a CRM configuration in which a cold, annular electron beam of radius $r_{b}$ propagates through a lossless, circular waveguide of radius $r_{w}$ in an axial magnetic field $B_{0}$. If the laboratory-frame dispersion relation for such a configuration is given by $D\left(k_{\|}, \omega\right)=0$, then the dispersion relation for a general reference frame moving in the axial direction with velocity $v$ (with respect to the laboratory frame) is given by

$$
D_{v}\left(k_{\|}^{\prime}, \omega^{\prime}\right)=D\left(k_{\|}\left(k_{\|}^{\prime}, \omega^{\prime}\right), \omega\left(k_{\|}^{\prime}, \omega^{\prime}\right)\right)=0
$$

In the above equation, $k_{\|}$and $\omega$ are the longitudinal wave number and the frequency relative to the laboratory frame. They are related to the corresponding general-frame quantities by the Lorentz transformation (Jackson 1975),

$$
\begin{gathered}
c k_{\|}=\gamma_{v}\left(c k_{\|}^{\prime}+\beta_{v} \omega^{\prime}\right), \\
\omega=\gamma_{v}\left(\omega^{\prime}+\beta_{v} c k_{\|}^{\prime}\right),
\end{gathered}
$$

where

$$
\begin{aligned}
& \beta_{v}=v / c, \\
& \gamma_{v}=\left(1-\beta_{v}^{2}\right)^{-\frac{1}{2}} .
\end{aligned}
$$

The CRM dispersion relation in the laboratory frame (for the case of a lossless waveguide) is discussed by Lau et al. (1981 a), and a detailed derivation is given by Chu \& Lin (1988). Applying the procedure in (1) to the laboratory-frame CRM dispersion relation, we find that the general-frame CMR dispersion relation describing the coupling of the $\mathrm{TE}_{m n}$ waveguide mode and the beam mode is given by

$$
\begin{aligned}
& \omega^{\prime 2}-c^{2} k_{\|}^{\prime 2}-\omega_{c}^{2}+\frac{4 N^{\prime} e^{2}}{\gamma^{\prime} m_{e} r_{\omega}^{2} K_{m n}}\left[\frac{\left(\omega^{2}-c^{2} k_{\|}^{\prime 2}\right) \beta_{\perp}^{2} H_{s m}\left(k_{m n} r_{b}, k_{m n} r_{L}\right)}{\left(\omega^{\prime}-k_{\|}^{\prime} v_{\|}^{\prime}-s \Omega_{c}^{\prime}\right)^{2}}\right. \\
& \left.-\frac{\left(\omega^{\prime}-k_{\|}^{\prime} v_{\|}^{\prime}\right) Q_{s m}\left(k_{m n} r_{b}, k_{m n} r_{L}\right)}{\left(\omega^{\prime}-k_{\|}^{\prime} v_{\|}^{\prime}-s \Omega_{c}^{\prime}\right)}\right]=0 .
\end{aligned}
$$


In the above equation,

$$
\begin{aligned}
& H_{s m}(x, y)= J_{s-m}^{2}(x) J_{s}^{\prime 2}(y) \\
& K_{m n}=J_{m}^{2}\left(\nu_{m n}\right)\left[1-m^{2} / \nu_{m n}^{2}\right] \\
& Q_{s m}(x, y)=2 H_{s m}(x, y)+y\left[J_{s-m}^{2}(x) J_{s}^{\prime}(y) J_{s}^{\prime \prime}(y)+\frac{1}{2} J_{s-m-1}^{2}(x) J_{s}^{\prime}(y) J_{s-1}^{\prime}(y)\right. \\
&\left.\quad-\frac{1}{2} J_{s-m+1}^{2}(x) J_{s}^{\prime}(y) J_{s+1}^{\prime}(y)\right] .
\end{aligned}
$$

Quantities appearing in (4) and (5) that are invariant under the Lorentz transformation are the waveguide radius $r_{w}$, the beam radius $r_{b}$, the waveguide-mode cutoff frequency $\omega_{c}$, and the electron Larmor radius $r_{L}=c \beta_{\perp}^{\prime} / \Omega_{c}^{\prime}$. The cutoff frequency is given by $\omega_{c}=c k_{m n}$ where $k_{m n}=\nu_{m n} / r_{w}$. The quantity $\nu_{m n}$ is the n-th nontrivial root of the first derivative of the Bessel function $J_{m}$. The quantities $\gamma^{\prime} m_{e} c^{2}, c \beta_{\perp}^{\prime}$, and $v_{\|}^{\prime}$ are the general-frame electron energy, transverse velocity, and longitudinal velocity, respectively. The relativistic electron cyclotron frequency is

$$
\Omega_{c}^{\prime}=\frac{c B_{0}}{\gamma^{\prime} m_{e} c}
$$

where $B_{0}$ is the (invariant) axial magnetic field. Finally, $N^{\prime}$ is the general-frame number of electrons per unit length in the axial direction.

Close to resonance both the uncoupled waveguide dielectric function $\left(\omega^{2}-c^{2} k_{\|}^{\prime 2}-\omega_{c}^{2}\right)$ and the beam dielectric function $\left(\omega^{\prime}-k_{\|}^{\prime} v_{\|}^{\prime}-s \Omega_{c}^{\prime}\right)$ are close to zero. Consequently, we neglect the second term in the square brackets in (4) and approximate the factor $\omega^{\prime 2}-c^{2} k^{\prime 2}$ in the first term by $\omega_{c}^{2}$. (Note that the ratio of the two terms in the square brackets is an invariant, so that the validity of this approximation is independent of the reference frame.) The resulting approximate dispersion relation can then be written in the following dimensionless form,

$$
D_{v}\left(\hat{k}^{\prime}, \hat{\omega}^{\prime}\right)=\left(\hat{\omega}^{\prime 2}-\hat{k}^{\prime 2}-1\right)\left(\hat{\omega}^{\prime}-\beta_{\|}^{\prime} \hat{k}^{\prime}-s b^{\prime}\right)^{2}+\epsilon^{\prime}=0
$$

where $\hat{\omega}^{\prime}=\omega^{\prime} / \omega_{c}$ and $\hat{k}^{\prime}=c k_{\|}^{\prime} / \omega_{c}=k_{\|}^{\prime} / k_{m n}$. The dimensionless magnetic field strength $b^{\prime}$ is given by

$$
b^{\prime}=\Omega_{c}^{\prime} / \omega_{c}
$$


Its general-frame value is related to the laboratory-frame value $(b)$ by

$$
b^{\prime}=b \gamma_{v}^{-1}\left(1-\beta_{v} \beta_{\|}\right)^{-1}
$$

The coupling constant $\epsilon^{\prime}$ is given by

$$
\epsilon^{\prime}=\frac{4 N^{\prime} e^{2} \beta_{\perp}^{2} H_{s m}\left(k_{m n} r_{b}, k_{m n} r_{L}\right)}{\gamma^{\prime} m r_{w}^{2} \omega_{c}^{2} K_{m n}}
$$

where the general-frame value is related to the laboratory-frame value $(\epsilon)$ by

$$
\epsilon^{\prime}=\epsilon \gamma_{v}^{-2}\left(1-\beta_{v} \beta_{\|}\right)^{-2}
$$




\section{PINCH POINTS IN THE GENERAL REFERENCE FRAME}

Consider a CRM mode which is initially in an equilibrium state with no radiation present. The normalized Green's function $\hat{G}(z=v t, t)=G(z=v t, t) / \omega_{c}$ giving the response of the mode to a delta-function disturbance $\delta(z) \delta(t)$ has the time-asymptotic behavior

$$
t^{-1} \ln \hat{G}(v t, t) \sim \gamma_{v}^{-1} \operatorname{Im}\left(\hat{\omega}_{s}^{\prime}\right)
$$

where $\hat{\omega}_{s}^{\prime}$ is the pinch-point coordinate for the general reference frame moving with the normalized velocity $\beta_{v}=v / c$ relative to the laboratory frame (Bers et al. 1984).

The conditions for a saddle point in the general reference frame are

$$
D_{v}\left(\hat{k}^{\prime}, \hat{\omega}^{\prime}\right)=0
$$

and

$$
\frac{\partial D_{v}\left(\hat{k}^{\prime}, \hat{\omega}^{\prime}\right)}{\partial \hat{k}^{\prime}}=0,
$$

(Briggs 1964; Bers 1983, 1984). Substituting (7) into (14), we obtain the saddle-point condition

$$
\hat{k}^{\prime}\left(\hat{\omega}^{\prime}-\beta_{\|}^{\prime} \hat{k}^{\prime}-s b^{\prime}\right)+\beta_{\|}^{\prime}\left(\hat{\omega}^{\prime 2}-\hat{k}^{\prime 2}-1\right)=0 .
$$

Then substituting (15) into (7), we obtain a second independent saddle-point condition

$$
\beta_{\|}^{\prime} \epsilon^{\prime}=\hat{k}^{\prime}\left(\hat{\omega}^{\prime}-\beta_{\|}^{\prime} \hat{k}^{\prime}-s b^{\prime}\right)^{3} .
$$

Saddle-point coordinates for the general frame are obtained by solving (15) and (16) simultaneously. However, in order that such a saddle point be a pinch point it is necessary that the saddle point be formed by the merging of an $L$-contour image from above the real $\hat{k}^{\prime}$-axis with one from below and that at the saddle point $\operatorname{Im}\left(\hat{\omega}^{\prime}\right)>0$.

For the case of $s b^{\prime}>1$, approximate pinch-point coordinates are obtained by writing

$$
\begin{aligned}
& \hat{k}^{\prime}=\hat{k}_{ \pm}^{\prime}+\delta \hat{k}_{ \pm}^{\prime}, \\
& \hat{\omega}^{\prime}=\hat{\omega}_{ \pm}^{\prime}+\delta \hat{\omega}_{ \pm}^{\prime},
\end{aligned}
$$


where $\hat{k}_{ \pm}^{\prime}$ and $\hat{\omega}_{ \pm}^{\prime}$ are the simultaneous solutions of the uncoupled waveguide dispersion relation

$$
\hat{\omega}^{2}-\hat{k}^{2}-1=0
$$

and beam dispersion relation

$$
\hat{\omega}^{\prime}-\beta_{\|} \hat{k}^{\prime}-s b=0
$$

These quantities are given by

$$
\begin{aligned}
& \hat{k}_{ \pm}^{\prime}=\frac{1}{b_{0}^{\prime 2}}\left[s b^{\prime} \beta_{\|}^{\prime} \pm\left(s^{2} b^{2}-b_{0}^{\prime 2}\right)^{\frac{1}{2}}\right] \\
& \hat{\omega}_{ \pm}^{\prime}=\beta_{\|}^{\prime} \hat{k}_{ \pm}^{\prime}+s b^{\prime},
\end{aligned}
$$

where

$$
b_{0}^{\prime}=\left(1-\beta_{\|}^{\prime 2}\right)^{\frac{1}{2}} .
$$

The quantity $b_{0}^{\prime}$ is called the grazing value of $b^{\prime}$ (Lau et al. $1981 a$ ). By substituting (17) into (15) and (16) and retaining only lowest order powers of $\delta \hat{k}_{ \pm}^{\prime}$ and $\delta \hat{\omega}_{ \pm}^{\prime}$, it is found that at a saddle point

$$
\begin{aligned}
\left(\delta \hat{\omega}_{ \pm}^{\prime}\right)^{3} & =-\frac{27}{8} \frac{\hat{k}_{ \pm}^{\prime 2} \beta_{\|}^{\prime} \epsilon^{\prime}}{\left(\beta_{\|}^{\prime} \hat{\omega}_{ \pm}^{\prime}-\hat{k}_{ \pm}^{\prime}\right)^{3}} \\
\delta \hat{k}_{ \pm}^{\prime} & =\frac{\left(\hat{k}_{ \pm}^{\prime}+2 \hat{\omega}_{ \pm}^{\prime} \beta_{\|}^{\prime}\right)}{3 \beta_{\|}^{\prime} \hat{k}_{ \pm}^{\prime}} \delta \hat{\omega}_{ \pm}^{\prime} .
\end{aligned}
$$

In the following analysis, $\delta \hat{\omega}_{+}^{\prime}$ and $\delta \hat{k}_{+}^{\prime}$ are referred to as upshifted solutions and to $\delta \hat{\omega}_{-}^{\prime}$ and $\delta \hat{k}_{-}^{\prime}$ as downshifted solutions.

It is necessary to investigate whether or not the approximate saddle points given by (22) are formed by the merging of an $L$-contour image from above the real $\hat{k}^{\prime}$ axis with one from below. Substituting (17) into the CRM dispersion relation in (7) and retaining only lowest powers of $\delta \hat{k}_{ \pm}^{\prime}$ and $\delta \hat{\omega}_{ \pm}^{\prime}$, we obtain the approximate dispersion relation

$$
2\left(\hat{\omega}_{ \pm}^{\prime} \delta \hat{\omega}_{ \pm}^{\prime}-\hat{k}_{ \pm}^{\prime} \delta \hat{k}_{ \pm}^{\prime}\right)\left(\delta \hat{\omega}_{ \pm}^{\prime}-\beta_{\|}^{\prime} \delta \hat{k}_{ \pm}^{\prime}\right)^{2}+\epsilon^{\prime}=0
$$


For large positive $\operatorname{Im}\left(\delta \hat{\omega}_{ \pm}^{\prime}\right)$, the solutions of (23) behave asymptotically as

$$
\begin{array}{ll}
\operatorname{Im}\left(\delta \hat{k}_{ \pm}^{\prime}\right) \sim \frac{\hat{\omega}_{ \pm}^{\prime}}{\hat{k}^{\prime} \pm} \operatorname{Im}\left(\delta \hat{\omega}_{ \pm}^{\prime}\right), & \quad(1 \text { solution }) \\
\operatorname{Im}\left(\delta \hat{k}_{ \pm}^{\prime}\right) \sim \frac{\operatorname{Im}\left(\delta \hat{\omega}_{ \pm}^{\prime}\right)}{\beta_{\|}^{\prime}}, & \quad(2 \text { solutions })
\end{array}
$$

Consequently, a pinch point can be can be formed only if $\beta_{\|}^{\prime}<0$ and $\hat{\omega}_{ \pm}^{\prime}$ and $\hat{k}_{ \pm}^{\prime}$ have the same sign, or if $\beta_{\|}^{\prime}>0$ and $\hat{\omega}_{ \pm}^{\prime}$ and $\hat{k}_{ \pm}^{\prime}$ have opposite signs. Using (20) it is straightforward to show that the upshifted solutions $\left(\delta \hat{k}_{+}^{\prime}, \delta \hat{\omega}_{+}^{\prime}\right)$ can give rise to pinch points only if $\beta_{\|}^{\prime}<0$ and $s b^{\prime}>1$. Moreover, the downshifted solutions can give rise to pinch points only if $\beta_{\|}^{\prime}>0$ and $s b^{\prime}>1$. Therefore, by choosing solutions in (22) for which $\operatorname{Im}\left(\delta \hat{\omega}_{ \pm}^{\prime}\right)>0$, the following approximate pinch-point coordinates are obtained,

$$
\begin{aligned}
\hat{\omega}_{s+}^{\prime} & =\hat{\omega}_{+}^{\prime}+\delta \hat{\omega}_{+}^{\prime} \\
& =\hat{\omega}_{+}^{\prime}+\frac{3}{4} \frac{\left(\hat{k}_{+}^{\prime 2}\left|\beta_{\|}^{\prime}\right| \epsilon^{\prime}\right)^{\frac{1}{3}}}{\left(\hat{k}_{+}^{\prime}-\beta_{\|}^{\prime} \hat{\omega}_{+}^{\prime}\right)}(1+i \sqrt{3}), \\
\hat{\omega}_{s-}^{\prime} & =\hat{\omega}_{-}^{\prime}+\delta \hat{\omega}_{-}^{\prime} \\
& =\hat{\omega}_{-}^{\prime}+\frac{3}{4} \frac{\left(\hat{k}_{-}^{\prime 2} \beta_{\|}^{\prime} \epsilon^{\prime}\right)^{\frac{1}{3}}}{\left(\beta_{\|}^{\prime} \hat{\omega}_{-}^{\prime}-\hat{k}_{-}^{\prime}\right)}(1+i \sqrt{3}),
\end{aligned}
$$

and

$$
\hat{k}_{s \pm}^{\prime}=\hat{k}_{ \pm}^{\prime}+\frac{\left(\hat{k}_{ \pm}^{\prime}+2 \beta_{\|}^{\prime} \hat{\omega}_{ \pm}^{\prime}\right)}{3 \beta_{\|}^{\prime} \hat{k}_{ \pm}^{\prime}} \delta \hat{\omega}_{ \pm}^{\prime} .
$$

The above equations are applicable only if $s b^{\prime}>1$. Equation (25) is to be used only if $\beta_{\|}^{\prime}<0$, and (26) is to be used only if $\beta_{\|}^{\prime}>0$. Approximate pulse shapes are obtained from (12), (25) and (26). The pulse obtained from (25) is referred to as the upshifted pulse and that obtained from (26) as the downshifted pulse.

The above analysis does not imply that pinch points do not exist for $s b^{\prime}<1$. It does imply that a power series expansion in $\delta \hat{k}_{ \pm}^{\prime}$ and $\delta \hat{\omega}_{ \pm}^{\prime}$ about $\hat{k}_{ \pm}^{\prime}$ and $\hat{\omega}_{ \pm}^{\prime}$ will not converge to the pinch point for $s b^{\prime}<1$. Instead, the power series converges to another saddle point which is not a pinch point.

We make some remarks concerning circumstances under which the laboratory-frame limit of (26) can be used to calculate approximate growth rates of absolute instabilities 
(i.e., circumstances under which $s b>1$ ). Ordinarily, a CRM is designed to generate or amplify radiation characteristic of the first beam harmonic $(s=1)$ and a selected waveguide mode referred to as the waveguide operating mode. The laboratory-frame value $b$ is usually chosen to be less than one to avoid the absolute instability in the operating mode that will result from a negative value of $\hat{k}$ at the downshifted intersection of the uncoupled, laboratory-frame dispersion relations for the waveguide mode (18) and the beam mode (19). This intersection is given by $\hat{k}_{-}$in the laboratory-frame limit of (20). Under these circumstances, (26) cannot be used to estimate the growth rate of any absolute instability which may still be present in the operating mode. On the other hand, (26) is useful for approximating growth rates of absolute instabilities associated with higher beam harmonics $(s>1)$. Moreover, if the CRM is operating in a high-frequency waveguide mode, (26) may be useful in approximating growth rates of absolute instabilities in some of the lower-frequency waveguide modes (e.g., an absolute instability in the $T E_{11}$ mode when operating in the $\mathrm{TE}_{01}$ mode).

Equations (25) and (26) are usually useful in estimating pulse shapes for the case of $s=1$ even when $b<1$. The behavior of $b^{\prime}$ as a function of $\beta_{v}$ is given in (9). Its value is zero when $\beta_{v}= \pm 1$, and it approaches a maximum value of $b / b_{0}$ at $\beta_{v}=\beta_{\|}$, where $b_{0}$ is defined in the laboratory-frame limit of (21). This maximum value is often greater than one. As a consequence, (25) and (26) often give very good approximations of the upshifted and downshifted pulse shapes in (12) over much of their $\beta_{v}$-intervals even though (26) may not give an approximation of the pulse height at $\beta_{v}=0$ (i.e., the absolute instability growth rate). Moreover, once (26) is used to provide initial values for determining exact pinch-point coordinates numerically for a reference frame with $\beta_{v}>0$, the resulting coordinates can be used as initial values for the numerical determination of pinch-point coordinates for reference frames with $s b^{\prime}<1$.

A more direct method of determining pinch-point coordinates $\left(\hat{k}_{s}^{\prime}, \hat{\omega}_{s}^{\prime}\right)$ numerically for the case of $s b^{\prime}<1$ is based on earlier work in which the critical value $\epsilon_{c}$ of the coupling constant $\epsilon$ for the onset of absolute instability was derived (Davies 1989). This work (carried out in the laboratory frame) is easily extended to the general reference frame, because the form of $(7)$ is the same for all frames. Subject to the rather weak condition 
[stated without primes in Davies (1989)] that

$$
\hat{\omega}_{s}^{\prime}-\hat{k}_{s}^{\prime} / \beta_{\|}^{\prime}>0
$$

a pinch point will be present in the general reference frame when the coupling constant $\epsilon^{\prime}$ in (7) obeys

$$
\epsilon^{\prime}>\epsilon_{c}^{\prime}
$$

where the critical coupling constant is given by

$$
\epsilon_{c}^{\prime}=27 \beta_{\|}^{\prime 2} \hat{k}_{s}^{\prime 4}
$$

At this critical value of $\epsilon^{\prime}$, the pinch point lies on the real $\hat{k}^{\prime}$-axis with the coordinate

$$
\hat{k}_{s}^{\prime}=\frac{1}{2}\left(1+8 \beta_{\|}^{\prime 2}\right)^{-1}\left\{-4 \beta_{\|}^{\prime} s b^{\prime}+\left[16 \beta_{\|}^{\prime 2} s^{2} b^{2}+2\left(1+8 \beta_{\|}^{\prime 2}\right)\left(1-s^{2} b^{\prime 2}\right)\right]^{\frac{1}{2}}\right\}
$$

The corresponding real frequency is

$$
\hat{\omega}_{s}^{\prime}=4 \beta_{\|}^{\prime} \hat{k}_{s}^{\prime}+s b^{\prime}
$$

The saddle point at the onset of becoming a pinch point is of third order (Davies 1989), so that no linear expansion about the saddle point exists to approximate pinch-point coordinates for the case of $\epsilon^{\prime}>\epsilon_{c}^{\prime}$. However, initial values for a numerical computation of pinch-point coordinates in this case are provided by $\hat{k}_{g}^{\prime}$ in (31) and $\hat{\omega}_{s}^{\prime}$ in (32) with a small positive imaginary part added to $\hat{\omega}_{s}^{\prime}$. We divide the interval between $\epsilon^{\prime}$ and $\epsilon_{c}^{\prime}$ into small increments, and (starting with $\epsilon_{c}^{\prime}$ ) successively compute the pinch-point coordinates after each increment using Newton's method to solve (15) and (16) simultaneously.

If the condition in (28) is not obeyed [with $\hat{k}_{s}^{\prime}$ and $\hat{\omega}_{s}^{\prime}$ defined in (31) and (32)], then the saddle point at the onset of becoming a pinch point is of second order. The generalframe saddle-point coordinates for the critical coupling constant are no longer given by (31) and (32). Instead,

$$
\begin{aligned}
& \hat{k}_{s}^{\prime}=\frac{\beta_{\|}^{\prime}}{4 b_{0}^{\prime 2}}\left[s b^{\prime}+\left(s^{2} b^{2}+8 b_{0}^{\prime 2}\right)^{\frac{1}{2}}\right] \\
& \hat{\omega}_{s}^{\prime}=\hat{k}_{s}^{\prime} / \beta_{\|}^{\prime} .
\end{aligned}
$$


The general-frame critical coupling constant is now given by

$$
\epsilon_{c}^{\prime}=\frac{k_{s}^{\prime}}{\beta_{\|}^{\prime 4}}\left(b_{0}^{\prime 2} k_{s}^{\prime}-\beta_{\|}^{\prime} s b^{\prime}\right)^{3} .
$$

Equations (33) and (34) now provide a starting point for computing pinch points for $\epsilon^{\prime}>\epsilon_{c}^{\prime}$. 


\section{EXAMPLES OF PULSE SHAPES}

Several of the numerical examples of pulse shapes presented in this section are relevant to present or proposed CARM experiments at M.I.T. (Pendergast et al. 1988; Danly et al. 1988, 1989). The physical properties of such experiments are usually given in terms of the frequency of the radiation to be generated or amplified, the beam kinetic energy, the beam current, the ratio of the perpendicular to the parallel electron velocity,

$$
\alpha=\beta_{\perp} / \beta_{\|}
$$

the waveguide and beam radii ( $r_{w}$ and $r_{b}$, respectively), and the detuning parameter $\Delta$. The detuning parameter (Bratman et al. 1981; Danly et al. 1988) is defined by

$$
\Delta=\frac{2\left(1-\beta_{\|} / \beta_{\phi}\right)}{\beta_{\perp}^{2}\left(1-\beta_{\phi}^{-2}\right)}\left[1-\beta_{\|} / \beta_{\phi}-b\left(1-\beta_{\phi}^{-2}\right)^{\frac{1}{2}}\right]
$$

where $\beta_{\phi}$ is the phase velocity of the waveguide radiation given by

$$
\beta_{\phi}=\left(1-\frac{c^{2} \nu_{m n}^{2}}{r_{w}^{2} \mathcal{L}^{2}}\right)^{-\frac{1}{2}}
$$

The value of $\Delta$ is proportional to the laboratory-frame value of the beam-mode dielectric function $\hat{\omega}-\beta_{\|} \hat{k}-1$. Once these physical properties are specified, laboratory-frame values of the parameters appearing in the CRM dispersion relation (7) are obtained as follows. The parameter $\beta_{\|}$is obtained from the beam kinetic energy and (34). The normalized magnetic field strength $b$ is obtained from (36), (37), and the specified radiation frequency. This calculation is carried out for a specified waveguide mode which we refer to as the waveguide operating mode. The laboratory-frame coupling constant $\epsilon$ is obtained from (10). A convenient expression for this constant is

$$
\epsilon=\frac{4 \beta_{\perp}^{2}}{\gamma \beta_{\|}} \frac{\left[J_{s-m}\left(k_{m n} r_{b}\right) J_{s}^{\prime}\left(k_{m n} r_{L}\right)\right]^{2}}{\left(\nu_{m n}^{2}-m^{2}\right) J_{m}^{2}\left(\nu_{m n}\right)} \frac{I}{I_{A}}
$$

where $I$ is the beam current and $I_{A}=m c^{3} / e=17.045 k A$. General frame values of $b^{\prime}$, $\epsilon^{\prime}$, and $\beta_{\|}^{\prime}$ are obtained respectively from (9), (11), and the well known velocity addition formula $\beta_{\|}^{\prime}=\left(\beta_{\|}-\beta_{v}\right) /\left(1-\beta_{\|} \beta_{v}\right)$. 


\subsection{First Harmonic and Operation in the Lowest Frequency Waveguide Mode}

For the first example, we consider parameters relevant to the $140 \mathrm{GHz}$ cyclotron autoresonance maser (CARM) experiment at M.I.T. Operation in the $\mathrm{TE}_{11}$ waveguide mode is considered with a beam current of $20 \mathrm{~A}$ and a beam kinetic energy of $450 \mathrm{kV}$. The ratio of the perpendicular to parallel electron velocity $\alpha$ is selected to be 0.4 . We choose a waveguide radius $r_{w}$ of $0.236 \mathrm{~cm}$, and for simplicity assume a beam radius of zero and a detuning $\Delta$ of zero. Corresponding laboratory-frame values of the parameters appearing in (7) are $\beta_{\|}=0.7864, b=0.9102$, and $\epsilon=0.9700 \times 10^{-4}$. We will consider the first beam harmonic (i.e., $s=1$ ).

Pulse shapes for this example are presented in figure 1. Exact pulse shapes [obtained by solving (15) and (16) numerically and applying (12)] are shown by solid lines. There are two pulses, the more rapidly moving upshifted pulse and the slower moving downshifted pulse. The upshifted pulse represents the growth in amplitude of the radiation usually desired from the CARM. The upshifted pulse is convective because it moves to the right away from the origin at $\beta_{v}=0$. On the other hand, the downshifted pulse represents an absolute instability because it encompasses the origin. In this example, it represents the growth of unwanted oscillations in the system. The growth rate of the absolute instability is given by the height of the downshifted pulse at the origin. This growth rate is seen to be small in comparison with the growth rate at the maximum of the upshifted pulse. The upshifted and downshifted pulses are not separated but join at a value of $\beta_{v}$ which is approximately equal to the laboratory-frame beam velocity $\beta_{\|}$. Although it is not evident from figure 1, finer scale computations show that the slope of the pulse-shape curve is continuous at this joining.

Approximate pulse shapes obtained from (25), (26), and (12) are shown by the solid squares in figure 1. These are seen to provide a good approximation of the exact pulse shapes over much of the $\beta_{v}$-interval of the pulses. As was discussed in $\S 3,(25)$ and (26) are not applicable if $s b^{\prime}<1$. Using (9) we find that $s b^{\prime}=b^{\prime}<1$ if $\beta_{v}<0.1230$ or if $\beta_{v}>0.9640$. It is evident from figure 1 that (25) and (26) do not give valid approximations of the pulse shapes for these ranges of $\beta_{v}$. In particular, we note that (26) does not provide an approximation for the growth rate of the absolute instability (i.e., the pulse height at 
$\left.\beta_{v}=0\right)$. The approximate expressions in (25) and (26) are also invalid for $\beta_{v}$ sufficiently close to the laboratory-frame beam velocity $\beta_{\|}$. It is evident that (25) and (26) both give a zero pulse height at $\beta_{v}=\beta_{\|}$, instead of the nonzero value given by the exact computations. [It is also evident from (22) that the expression for $\delta \hat{k}_{ \pm}^{\prime}$ at the pinch point diverges as $\beta_{v}$ approaches $\beta_{\|}$.]

In earlier work (Davies et al. 1988), it was shown that to each extremum in the pulse-shape curve there is a corresponding extremum of equal height in the laboratoryframe temporal growth-rate curve $[\operatorname{Im}(\hat{\omega})$ vs. real $\hat{k}]$. In figure 2 the growth-rate curve is presented for this example, obtained from the CRM dispersion relation in (7). The growthrate curves have two maxima (downshifted and upshifted) with a minimum in between. Comparing figures 1 and 2, it is evident that the upshifted [downshifted] pulse height maximum equals the upshifted [downshifted] growth-rate-curve maximum. The minimum in the pulse-shape curve, where the upshifted and downshifted pulses join, is equal to the minimum in the growth-rate curves. It is interesting that the joining of the pulses can be regarded to be a consequence of the joining of the growth-rate-curve maxima. Another interesting property of figures 1 and 2 is that absolute instability is evident in figure 1 even though figure 2 shows no instability for negative values of real $\hat{k}$ (i.e., there is no backward traveling unstable wave).

The downshifted pulse in figure 1 shows the presence of absolute instability because it encompasses the origin. By reducing the ratio $\alpha=\beta_{\perp} / \beta_{\|}$while leaving fixed the other physical properties given in the first paragraph of this section, we can bring the left edge of the pulse to the right of the origin. This change causes the the downshifted pulse to become convective. In figure 3 we show pulses for $\alpha=0.4,0.33$, and 0.3 . We see that a reduction of $\alpha$ from 0.4 to 0.33 causes the downshifted pulse to become convective but at the same time reduces the maximum of the upshifted pulse (and therefore the temporal growth rate of the amplitude of the desired radiation).

We next consider the pulse shape for the case where the axial magnetic field is reduced to its grazing value. Grazing refers to the situation in which the uncoupled waveguide and beam dispersion relations [(18) and (19)] intersect at a single tangent point on a graph of $\hat{\omega}$ vs. $\hat{k}$. For illustrative purposes, we choose the same waveguide operating mode, beam 
current, energy, and waveguide and beam radii as in the previous examples. In addition, $\alpha=\beta_{\perp} / \beta_{\|}=0.34$ is selected. Then $\beta_{\|}=0.8109$ and the grazing value of the dimensionless magnetic field $b$ is given by $b=b_{0}=\left(1-\beta_{\|}^{2}\right)^{\frac{1}{2}}=0.5852$. The laboratory-frame coupling constant [obtained from (38)] is given by $\epsilon=3.055 \times 10^{-4}$. The pulse-shape curve for this case is shown in figure 4. Evidently, the upshifted and downshifted pulses merge into a single pulse as $b$ is reduced to its grazing value. The instability is seen to be convective because both edges of the pulse move to the right away from the origin. Note that the left edge of the pulse is much further to the right than the left edges of the pulses in figures 1 or 3. The corresponding temporal growth-rate curve is shown in figure 5 . The reduction in $b$ to its grazing value causes the separate upshifted and downshifted growth-rate curve maxima to merge into a single maximum. In accordance with the theorem quoted earlier in this section, the single maximum in the pulse-shape curve in figure 4 equals the single maximum in the growth-rate curve in figure 5.

\subsection{Pulses for Higher Beam Harmonics}

CRM modes with $s b \geq 1$ will be absolutely unstable for all values of $\epsilon>0$ (Davies 1989). If $b \geq 0.5$ (which is usually the case), then all instabilities associated with beam harmonics above the fundamental $(s>1)$ will be absolute. Consequently, it is interesting to compare pulses for the fundamental mode with those for higher harmonics. As an example, we use the same physical properties as those used for the example in figure 1, except that we consider an annular beam of radius $r_{b}=0.18 \mathrm{~cm}$ and we choose $\alpha=\beta_{\perp} / \beta_{\|}=0.33$. With a detuning of zero and operation in the first harmonic of the $\mathrm{TE}_{11}$ mode, the corresponding laboratory-frame values of the parameters appearing in the dispersion relation in (7) are $b=0.8452, \beta_{\|}=0.8043$ and $\epsilon=1.997 \times 10^{-5}, 4.743 \times 10^{-7}$, and $4.364 \times 10^{-10}$ for the first three harmonics $(s=1-3)$, respectively. In figure 6 , pulse-shape curves are presented for these parameters for the first three beam harmonics. The exact curves are shown by the solid lines. As expected, the pulses for the higher harmonics encompass the origin. The growth rate of the absolute instability in the second harmonic (the pulse height at $\beta_{v}=0$ ) is seen to be a little more than a third of the growth rate of the convective instability in the first harmonic. Note that the absolute instability growth rates for both the second and third harmonics are only slightly less than the maxima of the corresponding 
downshifted pulses (or equivalently, the corresponding downshifted temporal growth-rate curve maxima). The solid squares in figure 6 show the approximate pulse shape for the second harmonic obtained from (25), (26), and (12). Note that they provide an excellent approximation to the exact curve over most of the interval of the pulse. In particular, (26) provides an excellent approximation of the growth rate of the absolute instability in the second harmonic.

\subsection{Operation in Higher Frequency Waveguide Modes}

We next investigate pulse shapes which result from operating in a higher frequency waveguide mode than the lowest frequency mode. Operation in such a mode usually causes absolute instabilities to be present in lower frequency modes. This situtation is understood by referring to figure 7 , which is a schematic plot of the uncoupled beam dispersion relation (19) and the uncoupled $T E_{13}$ and $T E_{11}$ waveguide dispersion relations (18) for the case of operation in the $\mathrm{TE}_{13}$ mode. The downshifted intersection of the beam-mode dispersion relation is shown to occur at positive $k$, indicating that absolute instability is not necessarily present in the $\mathrm{TE}_{13}$ mode. On the other hand, the downshifted intersection of the beam dispersion dispersion relation with the $T E_{11}$ waveguide dispersion relation occurs at negative $k$, indicating that absolute instability is present in the $\mathbf{T E}_{11}$ mode.

As the first numerical example of operation in a higher frequency waveguide mode, we consider operation in the $\mathrm{TE}_{13}$ waveguide mode. In this case, a diagram similar to figure 7 , but including all lower frequency waveguide modes, indicates that absolute instabilities may be present in the first harmonic of several lower frequency modes. For simplicity, it is assumed that the beam radius $r_{b}$ is zero. Then, the coupling constant in (38) vanishes for all but $\mathrm{TE}_{1 n}$ modes, so that the only lower frequency $\mathrm{TE}$ modes to be considered are the $\mathrm{TE}_{12}$ and $\mathrm{TE}_{11}$ modes. We consider the case of a frequency of $17.136 \mathrm{GHz}$, a beam energy of $1.2 \mathrm{MeV}$, a beam current of $500 \mathrm{~A}$, and a waveguide radius $r_{w}$ of $6 \mathrm{~cm}$. The ratio $\alpha=\beta_{\perp} / \beta_{\|}$is selected to be 0.6 . The applied magnetic field is selected to provide a

detuning $\Delta$ of 0.4 . From the value of the beam energy and $\alpha$, we find that $\beta_{\|}=0.8184$. Using (36) and (37), it is found that $b=0.5507$ for the $\mathrm{TE}_{13}$ mode. From the definition $b=\Omega_{c} / \omega_{c}=\Omega_{c} r_{w} / c \nu_{m n}$, the corresponding values of $b$ for the $\mathrm{TE}_{12}$ and $\mathrm{TE}_{11}$ modes 
are 0.8818 and 2.553 , respectively. From (38), the respective values of $\epsilon$ are $2.479 \times 10^{-4}$, $6.170 \times 10^{-4}$ and $3.102 \times 10^{-3}$ for the $\mathrm{TE}_{13}, \mathrm{TE}_{12}$, and $\mathrm{TE}_{11}$ modes. Exact pulse shapes for these modes [obtained numerically from (15), (16), and (12)] are presented in figure 8. All of the pulse amplitudes are normalized to the cutoff frequency of the $\mathrm{TE}_{13}$ mode. Note that there is only a single pulse for the $\mathrm{TE}_{13}$ mode and that this pulse is convective. The $\mathrm{TE}_{12}$ downshifted pulse shows absolute instability and is of greater amplitude than the $\mathrm{TE}_{13}$ pulse. However, because all but a small portion of the $\mathrm{TE}_{12}$ pulse travels to the right, the growth rate of the $\mathrm{TE}_{12}$ absolute instability is only about one-fourth the growth rate of the $\mathrm{TE}_{13}$ convective instability. The $\mathrm{TE}_{11}$ pulse has a growth rate of only about one-half that of the $\mathrm{TE}_{13}$ pulse. But the growth rate of the associated $\mathrm{TE}_{11}$ absolute instability is also approximately one-half that of the $\mathrm{TE}_{13}$ convective instability, because the maximum of the $T E_{11}$ downshifted pulse is close to $\beta_{v}=0$. The solid squares in figure 8 show approximate pulse shapes for the $T_{11}$ mode obtained from (25) and (26). The approximation is excellent over most of the $\beta_{v}$-interval of the $\mathrm{TE}_{11}$ pulses including $\beta_{v}=0$.

As a second example of operation in a higher frequency waveguide mode, we consider operation in the $\mathrm{TE}_{01}$ mode and the resulting absolute instability in the lower frequency $\mathrm{TE}_{11}$ mode. The physical properties of the system are $\gamma=2.5, \alpha=0.5$, beam current $=100$ $\mathrm{A}, r_{b}=0.48 r_{w}$, and $b=1.15 b_{0}$ for the $\mathrm{TE}_{01}$ mode. Corresponding parameters in the CRM dispersion relation in (7) are $\beta_{\|}=0.8198, b=0.6586[1.371]$, and $\epsilon=5.039 \times 10^{-6}$ $\left[3.680 \times 10^{-4}\right]$ for the $\mathrm{TE}_{01}\left[\mathrm{TE}_{11}\right]$ mode. The above values conform with parameters used by Lin, Lin, \& Chu (1988) in simulations of a CARM, both with and without an upshifted $\mathrm{TE}_{01}$ drive signal. Pulse shapes for these parameters are shown in figure 9. The downshifted $T E_{01}$ pulse is seen to be convective, whereas the downshifted $\mathrm{TE}_{11}$ pulse shows absolute instability. Moreover, the growth rate of the $\mathrm{TE}_{11}$ absolute instability is seen to be approximately equal to the maximum growth rate of the upshifted $\mathrm{TE}_{01}$ pulse (or equivalently, to the maximum of the $\mathrm{TE}_{01}$ upshifted temporal growth rate curve).

\subsection{Discussion of Examples}

In earlier work (Davies, Davidson \& Johnston 1988), pulse shapes were analized for the free electron laser (FEL). A qualitative difference between FEL and CRM pulses is that 
(for typical FEL parameters) downshifted and upshifted FEL pulses are separated. On the other hand, if two pulse maxima are present for the CRM (typical of the CARM), then they are joined. If the CRM is operated close to grazing (typical for the gyrotron), then the pulses are merged into a single pulse. This difference is a reflection of a corresponding difference between the FEL and CARM temporal growth-rate curves. A more important difference between FEL and CRM pulses is that for the FEL the downshifted pulse maximum is ordinarily much smaller than the upshifted pulse maximum. If two maxima are present in the CRM, the downshifted maximum is always greater than the upshifted maximum. (This fact follows from the corresponding relation between CRM temporal growth-rate curve maxima.) Consequently, absolute instability is of much greater concern in the CARM than in the FEL.

The linear theory used in this paper can be used to make predictions concerning CRM stability properties if it shows that no absolute instability exists, or if it shows that an insufficient number of $e$-folding times exist for an absolutely unstable mode to be of concern. Otherwise, a comparison with nonlinear analyses and experimental results is needed in order to gain a further understanding of the significance of the linear results. The example in figure 9 has been analyzed by Lin, Lin \& Chu (1988) using a PIC simulation code. They find that when a signal builds up from noise, the presence of the $T E_{11}$ absolute instability prevents any significant development of the $\mathrm{TE}_{01}$ mode radiation. If a $\mathrm{TE}_{01}$ mode drive signal is present initially, then they find that the signal is amplified initially, but that eventually it is suppressed by the growth of the $\mathrm{TE}_{11}$ absolute instability. Note that the growth rate of the absolute instability in figure 9 is a larger fraction of the growth rate of the desired convective pulse than in any of the other figures. In particular, the relative growth rates of the higher-harmonic absolute instabilities in figure 6 are much smaller. 


\section{EFFECTS OF WAVE-GUIDE RESISTANCE}

If the resistance of the waveguide wall is nonzero, then the laboratory frame CRM dispersion relation is

$$
\left\{\hat{\omega}^{2}-\hat{k}^{2}-\left[1-(i+1)\left(1+\frac{m^{2}}{\nu_{m n}^{2}-m^{2}} \hat{\omega}^{2}\right) \frac{\delta}{r_{w}}\right]\right\}\left(\hat{\omega}-\beta_{\|} \hat{k}-s b\right)^{2}+\epsilon=0
$$

where $\delta$ is the skin depth of the wall material for radiation of frequency $\omega$ (Chu \& Lin 1988). Equation (39) together with (13) and (14) will be used later in this section to analyze growth rates of absolute instabilities (equal to pulse heights at $\beta_{v}=0$ ).

\subsection{Effects of Waveguide-Wall Resistance on Pulse Shapes}

In order to study the qualitative effects of a nonzero waveguide-wall resistance on pulse shapes, we require the CRM dispersion relation in the general reference frame. For simplicity, we consider only the case of coupling to waveguide modes having azimuthal symmetry (i.e., $\mathrm{TE}_{\mathrm{on}}$ modes). Moreover, the simplifying assumption is made that in the laboratory frame the permittivity and permeability of the wall material are exactly one. It is assumed that in the laboratory frame the wall material is an Ohmic conductor, with the current density $\mathbf{J}$ and the electric field $\mathbf{E}$ related by

$$
\mathrm{J}=\sigma \mathbf{E}
$$

where $\sigma$ is the conductivity. The charge density is assumed to be zero in the laboratory frame. Using the Lorentz transformations for the current density and the electromagnetic field (Jackson 1975), we rewrite (40) in terms of the currents and fields observed in the general reference frame, which moves with normalized velocity $\boldsymbol{\beta}_{v}$ in the axial direction. The resulting equation is

$$
\mathbf{J}^{\prime}=\sigma \gamma_{v}\left(\mathbf{E}^{\prime}-\beta_{v} \times \mathbf{B}^{\prime}\right)
$$

It is evident from (41) that the general-frame conductivity $\sigma^{\prime}$ is related to the laboratoryframe conductivity by

$$
\sigma^{\prime}=\gamma_{v} \sigma
$$

A derivation of the CRM laboratory-frame dispersion relation that includes nonzero skindepth effects for $\mathrm{TE}_{0 n}$ waveguide modes is given by Lau, et al. (1981 b). Using (41) together 
with the simplifying assumptions made at the beginning of this section, it is straightforward to extend the above derivation to the general reference frame. The general-frame dispersion relation for $T E_{0 n}$ modes is

$$
\left\{\hat{\omega}^{2}-\hat{k}^{\prime 2}-\left[1-\frac{\delta}{r_{w}}(i+1)\right]\right\}\left(\hat{\omega}^{\prime}-\beta_{\|}^{\prime} \hat{k}^{\prime}-s b^{\prime}\right)^{2}+\epsilon^{\prime}=0 .
$$

In the derivation of (43) it is assumed that the waveguide-wall material is a good conductor obeying the laboratory-frame condition that

$$
\frac{\omega^{2}}{c^{2}}-k_{\|}^{2} \ll \frac{4 \pi \sigma \omega}{c} .
$$

From (2) and (44), the corresponding general-frame condition is

$$
\frac{\omega^{\prime 2}}{c^{2}}-k_{\|}^{\prime 2} \ll \frac{4 \pi \sigma^{\prime}}{c}\left(\omega^{\prime}+k_{\|}^{\prime} v\right) \text {. }
$$

The skin depth $\delta$ in (43) is an invariant given by

$$
\delta=c\left[2 \pi \sigma^{\prime}\left(\omega^{\prime}+k_{\|}^{\prime} v\right)\right]^{-\frac{1}{2}}=c[2 \pi \sigma \omega]^{-\frac{1}{2}} .
$$

From (13), (14), and the dispersion relation in (43), we obtain the independent saddlepoint conditions

$$
\begin{gathered}
\hat{k}^{\prime}\left(\hat{\omega}^{\prime}-\beta_{\|}^{\prime} \hat{k}^{\prime}-s b^{\prime}\right)+\beta_{\|}^{\prime}\left\{\hat{\omega}^{\prime 2}-\hat{k}^{\prime 2}-\left[1-\frac{\delta}{r_{w}}(i+1)\right]\right\}=0, \\
\beta_{\|}^{\prime} \epsilon^{\prime}=\hat{k}^{\prime}\left(\hat{\omega}^{\prime}-\beta_{\|}^{\prime} \hat{k}^{\prime}-s b^{\prime}\right)^{3} .
\end{gathered}
$$

Effects of a nonzero skin depth on pulse shapes are obtained from (47). As an example, consider the first harmonic for a $\mathrm{TE}_{01}$ mode. The beam current is $300 \mathrm{~A}, \gamma=2.5, \alpha=0.5$, $r_{b}=0.48 r_{w}$, and $b=1.5 b_{0}=0.8591$. The corresponding value of $\beta_{\|}$is 0.8198 , and the value of $\epsilon$, obtained from (38) is $1.512 \times 10^{-4}$. Pulses for this example for various values of $\delta / r_{w}$ are shown in figure 10 . Notice that for $\delta / r_{w}=0$ a very slowly growing absolute instability is present, because the left edge of the pulse is slightly to the left of $\beta_{v}=0$. An increase in the value of $\delta / r_{w}$ to 0.005 causes the left edge of the pulse to move to the right of $\beta_{v}=0$. Consequently, the downshifted pulse becomes convective. 


\subsection{Effects of Waveguide-Wall Resistance on Absolute Instability Growth Rates}

In this section, the effects of wall resistance on the growth rates of absolute instabilities in $T E_{m n}$ modes are studied for arbitrary values of $m$. The analysis in this section is limited to the case of $0.5 \leq \beta_{\|}<1$. This is the region of interest for the CARM. (In the Appendix, a short discussion is presented of the effects of wall resistance in the small $\beta_{\|}$regime.) Since the growth rate of an absolute instability is the pulse height at $\beta_{v}=0$, we require only the laboratory-frame dispersion relation in (39). Practical values of $\delta / r_{w}$ range from zero to 0.01 . In the example in figure 10 , the absolute instability was suppressed by a realistic value of $\delta / r_{w}$. However, such suppression is not always possible. Considerable insight into the possibility of eliminating absolute instabilities by introducing a nonzero skin depth can be gained by referring to figure 11. This figure contains plots of the critical coupling constant $\epsilon_{c}$ required for absolute instability as a function of $\beta_{\|}$for various values of the quantity $s b$. A CRM mode will be absolutely unstable if its parameter point $\left(\beta_{\|}, \epsilon\right)$ lies above the $\epsilon_{c}$-contour corresponding to its value of $s b$. Contours are shown for two cases: $\delta / r_{w}=0$ and the extreme practical value $\delta / r_{w}=0.01$. The contours for $\delta / r_{w}=0$ are obtained analytically (Davies 1989). For these contours, $\epsilon_{c}$ approaches zero as $s b$ approaches one for any value of $\beta_{\|}$. The contours for $\delta / r_{w}=0.01$ apply to the case of $m=0$ in (39) (i.e., to $\mathrm{TE}_{\mathrm{On}}$ waveguide modes). These contours are obtained numerically by selecting a value of $\epsilon$ above that required for absolute instability and then reducing the value of $\epsilon$ until $\operatorname{Im}(\hat{\omega})$ at the pinch point obtained from (38), (13) and (14) reaches zero. Practically, the suppression of an absolutely unstable mode by wall resistance may be possible if its parameter point $\left(\beta_{\|}, \epsilon\right)$ lies below the $\epsilon_{e}$-contour corresponding to its value of $s b$ for $\delta / r_{w}=0.01$. For instance, the parameter point $\left(\beta_{\|}=0.8198\right.$ and $\left.\epsilon=1.512 \times 10^{-4}\right)$ of the example in figure 10 is located at $\mathrm{P}$ in figure 11 . The value of $s b=b$ for this example is 0.8591 . It is evident that for $\delta / r_{w}=0$ the point $\mathrm{P}$ lies above the $\epsilon_{c}$-contour for $s b=0.8591$, indicating that the mode is absolutely unstable. For the case of $\delta / r_{w}=0.01$, the position of the contour for $s b=0.8591$ can be estimated by extrapolating between the contours for $s b=0.85$ and 0.90 . It is evident that $\mathrm{P}$ lies below this extrapolated contour, indicating that the mode is absolutely stable when $\delta / r_{w}=0.01$.

Contours have also been computed for $\delta / r_{w}=0.01$ and $m=1$. For a given value of 
$s b$, the contour for $m=1$ lies above that for $m=0$. However, the contours for $m=0$ and $m=1$ are qualitatively similar. Consequently, figure 11 will be used in qualitative discussions of examples presented later in this section even though in some cases the waveguide modes involved are $\mathrm{TE}_{1 n}$.

For the case of $s b>1$ and $\delta / r_{w}>0$, it is not difficult to obtain a simple, approximate analytical expression for contours such as those in figure 11. In this case, the treatment used to obtain (26) is easily extended to determine approximate laboratory-frame pinch-point coordinates for the case of a small nonzero skin depth. By regarding $\delta \hat{\omega}_{-}, \delta \hat{k}_{-}$, and $\delta / r_{w}$ as small quantities, and employing (39) in (13) and (14), we find that the generalization of the laboratory-frame version of (26) is $\hat{\omega}_{s}=\hat{\omega}_{-}+\delta \hat{\omega}_{-}$where $\hat{\omega}_{-}$is given by $(20)$, and

$$
\begin{aligned}
& \operatorname{Re}\left(\delta \hat{\omega}_{-}\right)=\frac{1}{2}\left(\beta_{\|} \hat{\omega}_{-}-\hat{k}_{-}\right)^{-1}\left[\frac{3}{2}\left(\hat{k}_{-}^{2} \beta_{\| \epsilon}\right)^{\frac{1}{3}}-\beta_{\|}\left(1+\frac{m^{2}}{\nu_{m n}^{2}-m^{2}} \hat{\omega}_{-}^{2}\right) \frac{\delta}{r_{w}}\right]
\end{aligned}
$$

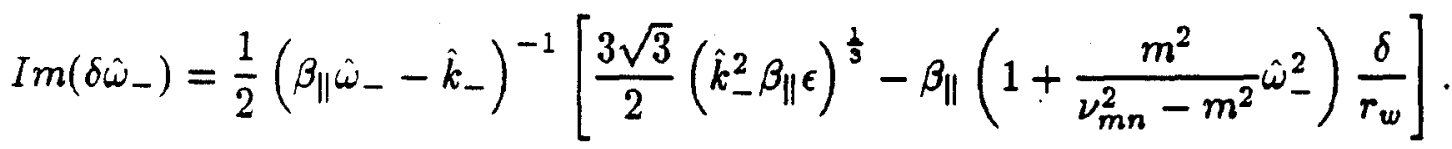

The corresponding approximate $\hat{k}_{3}$ is given by $\hat{k}_{s}=\hat{k}_{-}+\delta \hat{k}_{-}$, where $\hat{k}_{-}$is given by (20) and

$$
\delta \hat{k}_{-}=\left(3 \beta_{\|} \hat{k}_{-}\right)^{-1}\left[\left(\hat{k}_{-}+2 \beta_{\|} \hat{\omega}_{-}\right) \delta \hat{\omega}_{-}+\beta_{\|}(i+1)\left(1+\frac{m^{2}}{\nu_{m n}^{2}-m^{2}} \hat{\omega}_{-}^{2}\right) \frac{\delta}{r_{w}}\right] .
$$

By setting $\operatorname{Im}\left(\delta \hat{\omega}_{-}\right)=0$ in (48), we obtain the following approximation for the critical coupling constant $\epsilon_{c}$ for absolute instability:

$$
\epsilon_{c}=\frac{8}{81 \sqrt{3}} \frac{\beta_{\|}^{2}}{\hat{k}_{-}^{2}}\left(1+\frac{m^{2}}{\nu_{m n}^{2}-m^{2}} \hat{\omega}_{-}^{2}\right)^{3}\left(\frac{\delta}{r_{w}}\right)^{3} .
$$

Equation (50) is applicable only if $s b>1$. Approximate contours obtained from (50) and exact contours are shown in figure 12 for the case of $m=0$. It is seen that (50) gives excellent results for $s b>1.2$. Similar results are found for $m>0$.

As was discussed in \$3, approximate formulas have not been derived for pinch-point coordinates for the case of $s b<1$. However, it is not difficult to calculate approximate finite skin-depth corrections (for arbitrary values of $s b$ ) to numerically computed pinchpoint coordinates for the case of $\delta / r_{w}=0$. Let $\hat{\omega}_{s}=\hat{\omega}_{0}+\delta \hat{\omega}$ and $\hat{k}_{s}=\hat{k}_{0}+\delta \hat{k}$, where $\hat{\omega}$, 
and $\hat{k}_{s}$ are pinch-point coordinates for $\delta / r_{w}>0$, and $\hat{\omega}_{0}$ and $\hat{k}_{0}$ are previously computed pinch-point coordinates for the case of $\delta / r_{w}=0$. [See discussion in §3.] Using (39) in (13) and (14) and retaining only first-order terms in $\delta / r_{w}, \delta \hat{\omega}$, and $\delta \hat{k}$, the following approximate pinch-point coordinates are obtained:

$$
\begin{aligned}
& \hat{k}_{s}=\hat{k}_{0}-\frac{3 \delta}{2 r_{w}} \frac{\hat{k}_{0} \beta_{\|}(1+i)\left(1+\frac{m^{2}}{\nu_{m n}^{2}-m^{2}} \hat{\omega}_{0}^{2}\right)}{\left(\hat{k}_{0}-\beta_{\|} \hat{\omega}_{0}\right)\left(\hat{\omega}_{0}-4 \beta_{\|} \hat{k}_{0}-s b\right)}, \\
& \hat{\omega}_{s}=\hat{\omega}_{0}+\frac{\delta}{2 r_{w}} \frac{\beta_{\|}(1+i)\left(1+\frac{m^{2}}{\nu_{m n}^{2}-m^{2}} \hat{\omega}_{0}^{2}\right)}{\left(\hat{k}_{0}-\beta_{\|} \hat{\omega}_{0}\right)} .
\end{aligned}
$$

By setting $\operatorname{Im}\left(\hat{\omega}_{s}\right)=0$ in $(51)$, we obtain the following approximate value for the skin depth required to suppress an absolute instability:

$$
\frac{\delta}{r_{w}}=-\frac{2}{\beta_{\|}} \frac{\operatorname{Im}\left(\hat{\omega}_{0}\right)}{\operatorname{Im}\left[(1+i)\left(1+\frac{m^{2}}{\nu_{m n}^{2}-m^{2}} \hat{\omega}_{0}^{2}\right)\left(\hat{k}_{0}-\beta_{\|} \hat{\omega}_{0}\right)^{-1}\right]} .
$$

In examples presented below, the results obtained from (51) are compared with numerically computed exact results. In the Appendix, limitations on the use of (52) in the very small $\beta_{\|}$regime are discussed.

A question related to that of stabilizing absolute instabilities is that of reducing their growth rates by increasing $\delta / r_{w}$. We have not developed general rules relevant to this question. However, we present two illuminating examples. In table 1 the growth rate is plotted as a function of $\delta / r_{w}$ for the absolute instability in the second harmonic of the $\mathrm{TE}_{11}$ mode in figure 6 . The exact growth rates shown in column 2 of the table are obtained by substituting (39) into (13) and (14) and solving numerically for the pinch-point coordinates. Approximate growth rates, obtained from (51) and previously computed pinch-point coordinates for $\delta / r_{w}=0$, are shown in column 3 of the table. The approximation is seen to be excellent. Note from table 1 that, although the second-harmonic absolute instability is not stabilized, increasing $\delta / r_{w}$ from zero to 0.01 substantially reduces its growth rate. For this mode $s b=1.690$. The parameter point for this mode $\left(\beta_{\|}=0.8043\right.$ and $\left.\epsilon=4.743 \times 10^{-7}\right)$ is located at point $\mathrm{A}$ in figure 11. Point $\mathrm{A}$ is not very far above the contour for $s b=1.690$ 
for the case of $\delta / r_{w}=0.01$. The dimensionless coupling constant $\epsilon$ for the second harmonic for the $\mathrm{TE}_{11}$ mode $\left(4.74 \times 10^{-7}\right)$ is much smaller than that for the first harmonic $\left(2.00 \times 10^{-5}\right)$. As a result the former's parameter point is located near the bottom of figure 11 in the region occupied by $\epsilon_{c}$-contours with $s b>1$. The relatively small value of $\epsilon$ and resulting sensitivity of higher harmonic absolute instability growth rates to the value of $\delta / r_{w}$ in this example is primarily due to the factor $\left[J_{s}^{\prime}\left(k_{m n} r_{L}\right]^{2}=\left[J_{s}^{\prime}\left(\alpha \beta_{\|} / b\right)\right]^{2}\right.$ in the laboratory-frame expression for $\epsilon$ in (38). This factor decreases rapidly with increasing $s$. The small $x$ behavior of $J_{s}^{\prime}(x)$ is $x^{s-1} / 2^{s}(s-1)$ !

As a second example of the sensitivity of absolute instability growth rates to the value of $\delta / r_{w}$, we consider the modes in figure 8 . The figure shows absolute instability in the $\mathrm{TE}_{12}$ and $\mathrm{TE}_{11}$ modes due to operation in the $\mathrm{TE}_{13}$ mode. Both exact and approximate growth rates for the $\mathrm{TE}_{11}$ absolute instability as a function of $\delta / r_{w}$ are shown in table 2. Again the approximation given by (51) is seen to be excellent. An examination of table 2 shows that the decrease in growth rate of the of the $\mathrm{TE}_{11}$ mode absolute instability with increasing $\delta / r_{w}$ over the range zero to 0.01 is insignificant. A qualitative explaination for this weak $\delta / r_{w}$-dependence based on figure 11 is the following. The dimensionless magnetic field strength $b$ in (7) is given by $\Omega_{c} / \omega_{c}$, where $\omega_{c}$ is the cutoff frequency for the waveguide mode under consideration. The value of $b$ for the $T E_{13}$ mode is 0.5507 . However, because of the much lower $T E_{11}$ cutoff frequency, $b$ for the lower frequency $T E_{11}$ is has the very large value of 2.553. The dimensionless coupling constant $\epsilon$ for the $\mathrm{TE}_{11}$ mode $\left(3.102 \times 10^{-3}\right)$ is significantly larger than that for the $\mathrm{TE}_{13}$ operating mode $\left(\epsilon=2.479 \times 10^{-4}\right)$. This fact is primarily due to the increase in the denominator $\left(\nu_{1 n}^{2}-1\right) J_{1}^{2}\left(\nu_{1 n}\right)$ in (38) with increasing $n$. [The maxima of the oscillatory function $J_{1}^{2}\left(\nu_{1 n}\right)$ decrease with increasing $n$, but the function $\left(\nu_{1 n}^{2}-1\right)$ increases more rapidly.] As a consequence, the parameter point $\mathrm{B}$ for the $\mathrm{TE}_{11}$ mode is situated near the top of figure 11, far above the $\epsilon_{\mathrm{c}}$-contours of large $s b>1$ for $\delta / r_{w}=0.01$. Approximate and exact growth rates for the $\mathrm{TE}_{12}$ absolute instability are presented in table 3 . The growth rate of the $\mathrm{TE}_{12}$ absolute instability falls off slowly with increasing $\delta / r_{w}$, but nevertheless much more rapidly than that of the $\mathrm{TE}_{11}$ absolute instability. The parameter point for the $\mathrm{TE}_{12}$ mode is located at point $\mathrm{C}$ in figure 11. This point is above the $\epsilon_{c}$-contour for this mode $(s b=0.8818)$. However, an 
increase of $\delta / r_{w}$ from zero to 0.01 moves this contour significantly closer to point C. If the waveguide radius in this example is reduced from $r_{w}=6 \mathrm{~cm}$ to $r_{w}=5 \mathrm{~cm}$ while the remaining physical properties of the system remain fixed (see \$4.3), then the normalized $\mathrm{TE}_{12}$ absolute instability growth rate falls off rapidly from 0.005390 for $\delta / r_{w}=0$ to 0.0006843 for $\delta / r_{w}=0.01$. However, the decrease in the normalized growth rate of the $\mathrm{TE}_{11}$ absolute instability is still insignificant (from 0.08049 when $\delta / r_{w}=0$ to 0.07706 when $\left.\delta / r_{w}=0.01\right)$. 


\section{CONCLUSIONS}

In this paper, we have analyzed pulse shapes, which give the linear responces of cyclotron-resonance-maser (CRM) modes to a delta-function disturbance in time and space. For applied magnetic fields of strengths sufficiently above the grazing value, there are two pulses: an upshifted pulse, which is convective, and a downshifted pulse, which may be convective or which may encompass the point $\beta_{v}=0$, indicating absolute instability. The pulses are joined together at a nonzero minimum at a value of $\beta_{v}$ close to the longitudinal beam velocity $\beta_{\|}$. For magnetic fields close to or below the grazing value, the pulses are merged into a single pulse. There is a precise relationship between extrema of the pulse-shape curves and extrema of the temporal-growth-rate curves $[\operatorname{Im}(\hat{\omega})$ vs. real $h k]$. To each maximum in the pulse shape curve there is a corresponding maximum of equal height in the growth-rate curve. If a minimum exists in the pulse shape curve between the two maxima, then its value equals that of a corresponding minimum between the peaks of the growth-rate curves.

Pulse shapes for the CRM differ from those for the free-electron laser (FEL) in two respects. In normal operating regimes, the downshifted and upshifted pulses for the FEL are separated not joined or merged as they are for the CRM. Moreover, for most choices of FEL parameters, the FEL upshifted pulse has a much greater amplitude than the downshifted pulse. In the case of the CRM, if two pulses are present (typical for the CARM), then the downshifted pulse is of greater amplitude than the upshifted pulse. Consequently, absolute instability is of much greater concern in the CARM than in the FEL.

Several examples of pulse-shape curves have been presented. The examples illustrate absolute instability in the waveguide operating mode, absolute instability in higher harmonics of the operating mode, and absolute instability in lower-frequency waveguide modes when the operating mode is a higher-order waveguide mode. In these examples, methods of suppressing absolute instabilities cause the left edge of the pulse to move from negative to positive values of $\beta_{v}$ (i.e., cause the pulse to become a convective pulse propagating in the beam direction). 
Techniques for determining pinch-point coordinates in the general reference frame (including of course the laboratory frame) have been presented for the case of zero waveguidewall resistance. For the case of $s b^{\prime}>1$, approximate pinch-point coordinates are given by (25)-(27). Approximate expressions for pinch-point coordinates have not been developed for the case of $s b^{\prime}<1$. However, we have presented a straighforward method of determining such coordinates numerically. Equations (28)-(34) give exact values for the saddle-point coordinates and the critical coupling constant at the onset of the saddle point becoming a pinch point. By starting with such an initial pinch point it is straightforward to compute pinch-point coordinates for $\epsilon>\epsilon_{c}$, using methods discussed in $\S 3$.

The effects of introducing a nonzero wall resistance have been considered. Effects of wall resistance on pulse shapes have been analyzed for the special case of $\mathrm{TE}_{0 n}$ waveguide modes. Except for very small values of $\beta_{\|}$, increasing the wall resistance moves the left edge of the pulse towards larger values of $\beta_{v}$. If the pulse edge is moved across the point $\beta_{v}=0$, then absolute instability is suppressed. We have also considered the effectiveness of wall resistance in suppressing or reducing growth rates of absolute instabilities for $\mathrm{TE}_{m n}$ modes for general values of $m$. Approximate laboratory frame pinch-point coordinates for small $\delta / r_{w}$ and $s b>1$ are given in (48) and (49). Finite skin-depth corrections to pinch-point coordinates previously determined for $\delta / r_{w}=0$ are given in (51). We have presented examples which suggest that wall resistance will often be an effective method of suppressing (or reducing growth rates of) absolute instabilities in higher harmonics of the waveguide operating mode, because ordinarily the dimensionless coupling constant $\epsilon$ is very small for higher-harmonic modes. (See figure 11.) On the other hand, wall resistance will often have an insignificant effect on growth rates of absolute instabilities in some of the lower-frequency waveguide modes when the operating mode is a higher-order waveguide mode. The reason is that the dimensionless applied magnetic field $b=\Omega_{c} / \omega_{c}$ may be very large for lower-frequency (i.e., smaller- $\omega_{c}$ ) modes, and the dimensionless coupling constant $\epsilon$ for a lower-frequency mode will ordinarly be larger than that for the operating mode. In the Appendix, we point out that, for very small values of the axial beam velocity, wall resistance is destabilizing rather than stabilizing.

Finally, it has been pointed out that the significance of the linear results in this paper 
can be better understood only after a comparison with nonlinear and experimental results. In the case of an oscillator, it is important to know whether or not the growth of a slowlygrowing absolutely-unstable mode will be suppressed by the growth of a rapidly-growing convective mode having the desired frequency. In the case of an amplifier, it is important to know whether or not an input signal will suppress the growths of unwanted absolutelyunstable modes. 


\section{ACKNOWLEDGEMENTS}

The authors wish to thank B. R. Danly for many helpful discussions. This work was supported in part by the Department of Energy High Energy Physics Division, the Naval Research Laboratory Plasma Physics Division, and the Office of Naval Research. 


\section{REFERENCES}

Bers, A. 1983 Handbook of Plasma Physics, vol. 1. Basic Plasma Physics 1 (ed. A. A. Galeev \& R. N. Sudan), chap. 3.2. North-Holland.

Bers, A., Ram, A. K. \& Francis, G. 1984 Phys. Rev. Lett. 53, 1457.

Bratman, V. L., Ginzburg, N. S., Nusinovich, G. S., Petelin, M. I. \& Strelkov, P. S. 1981 Int. J. Electron. 51, 541.

Briggs, R. J. 1964 Electron-Stream Interactions with Plasmas. MIT Press.

Chu, K. R. \& Lin, A. T. 1988 IEEE Trans. Plasma Science, 16, 90.

Danly, B. G., Pendergast, K. D., Temkin, R. J. \& Davies, J. A. 1988 Proc. SPIE, 873, 143.

Danly, B. G., Davies, J. A., Pendergast, K. D., Temkin, R. J. \& Wurtele, J. S. 1989 Proc. SPIE, 1061.

Davies, J. A., Davidson, R. C. \& Johnston, G. L. 1988 J. Plasma Phys. 40, 1.

Fliflet, A. W. 1986 Int. J. Electron. 61, 1049.

Flyagin, V. A., Gaponov, A. V., Petelin, M. I. \& Yulpatov, V. K. 1977 IEEE Trans. Microwave Theory Tech. 25, 514.

Jackson, J. D. 1975 Classical Electrodynamics. Wiley.

Lau, Y. Y., Chu, K. R., Barnett, L. R. \& Granatstein, V. L. 1981 a Int. J. Infrared Millimeter Waves, $2,373$.

Lau, Y. Y., Chu, K. R., Barnett, L. R. \& Granatstein, V. L. 1981 b Int. J. Infrared Millimeter Waves, 2, 395.

Lin, A. T., Chu, K. R. \& Bromborsky A. 1987 IEEE Trans. Electron Devices, ED-34, 2621.

Lin, A. T., Lin, C. C. \& Chu, K. R. 1988 preprint.

Pendergast, K. D., Danly, B. G., Temkin, R. J. \& Wurtele, J. S. 1988 IEEE Trans. on Plasma Sci. PS-16, 122.

Schneider, J. 1959 Phys. Rev. Lett. 2, 504. 


\section{APPENDIX. INSTABILITY FOR SMALL AXIAL VELOCITY}

For very small values of $\beta_{\|}$and $s b<1$, the introduction of a waveguide-wall resistance is destabilizing rather than stabilizing. (We remark that such axial velocities are smaller than those usually employed even in the case of the gyrotron.) This behavior can be seen by referring to figure 13 . Figure 13 is similar to figure 11 , but it shows contours of the critical coupling constant over the entire range of $\beta_{\|}$from 0 to 1 for $\delta / r_{w}=0$ and 0.01 . For most of the range of $\beta_{\|}$, the contours for $\delta / r_{w}=0.01$ lie above corresponding contours for $\delta / r_{w}=0$, showing the stabilizing effect of the wall resistance. However, for sufficiently small values of $\beta_{\|}$, the contours for $\delta / r_{w}=0.01$ drop below the corresponding contours for $\delta / r_{w}=0$, indicating that the wall resistance is destabilizing. Also shown in the figure is the curve $A B$ upon which $\hat{\omega}_{s}-\hat{k}_{s} / \beta_{\|}=0$, where $\hat{\omega}_{s}$ and $\hat{k}_{s}$ are given by the laboratory frame limits of (31) and (32). In the laboratory frame, the condition in (28) is obeyed to the right of $A B$ and is disobeyed to the left of $A B$. From figure 13, the region in which wall resistance is destabilizing is seen to coincide approximately with the region to the left of $A B$.

It can be shown that $\epsilon_{c}$ approaches zero as $\beta_{\|}$approaches zero if $\delta / r_{w}>0$. Consequently, for any value of $s b$, the contour of critical $\epsilon$ for $\delta / r_{w}=0.01$ approaches zero in figure 13 as $\beta_{\|}$approaches zero. [An outline of the proof of the above statement is the following. From (33) and (34), it is seen that the critical coupling constant $\epsilon_{c}$ approaches a positive value as $\beta_{\|}$approaches zero, if $\delta / r_{w}=0$. Consider the saddle points given by (13) and (14) when $\beta_{\|}=0$ and $\epsilon>0$ is less than $\epsilon_{c}$ for $\delta / r_{w}=0$. A detailed analysis of (13), (14), and (39) shows that a small increment of $\delta / r_{w}$ above zero gives to one of these saddle points a small positive value of $\operatorname{Im}\left(\hat{\omega}_{s}\right)$, converting it into a pinch point. It follows that whenever $\delta / r_{w}>0, \epsilon_{c}$ approaches zero as $\beta_{\|}$approaches zero. This behavior is alse seen in the approximate formula for $\epsilon_{c}$ in (50) for the case of $s b>1$.]

The behavior discussed above places a restriction on the use of the approximation in (52). Numerical computations show that, for $s b<1$ and at sufficiently small values of $\beta_{\|},(52)$ gives negative values of $\delta / r_{w}$, reflecting the destabilizing effect of wall resistance at small $\beta_{\|}$. As this small $\beta_{\|}$region is approached from above (at constant $\epsilon$ ), $\delta / r_{w}$ 
approaches infinity at an asymptote separating the regions of positive and negative $\delta / r_{w}$. Since negative values of $\delta / r_{w}$ are unphysical and because of the assumption of small $\delta / r_{w}$ made in the derivation of (52), a result obtained from (52) is to be considered as valid only when it yields positive $\delta / r_{w} \ll 1$. 


\section{TABLES}

\begin{tabular}{ccc}
\hline$\delta / r_{w}$ & $\operatorname{Im}(\hat{\omega})$ Exact & $\operatorname{Im}(\hat{\omega})$ Approximate \\
\hline 0 & 0.004380 & 0.004380 \\
0.002 & 0.003577 & 0.003577 \\
0.004 & 0.002777 & 0.002773 \\
0.006 & 0.001978 & 0.001970 \\
0.008 & 0.001182 & 0.001167 \\
0.010 & 0.0003872 & 0.0003633 \\
\hline
\end{tabular}

Table 1: Growth rate of the absolute instability in the second beam harmonic for the $\mathrm{TE}_{11}$ mode in figure 6 as a function of $\delta / r_{w}$. Exact growth rates appear in column 2 , and approximate growth rates obtained from (51) appear in column 3.

\begin{tabular}{ccc}
\hline$\delta / r_{w}$ & $\operatorname{Im}(\hat{\omega})$ Exact & $\operatorname{Im}(\hat{\omega})$ Approximate \\
\hline 0 & 0.07972 & 0.07972 \\
0.002 & 0.07904 & 0.07904 \\
0.004 & 0.07837 & 0.07837 \\
0.006 & 0.07769 & 0.07769 \\
0.008 & 0.07702 & 0.07701 \\
0.010 & 0.07635 & 0.07633 \\
\hline
\end{tabular}

Table 2: Growth rate of the absolute instability in the $\mathrm{TE}_{11}$ mode in figure 8 as a function of $\delta / r_{w}$. Exact growth rates appear in column 2, and approximate growth rates obtained from (51) appear in column 3. 


\begin{tabular}{ccc}
\hline$\delta / r_{w}$ & $\operatorname{Im}(\hat{\omega})$ Exact & $\operatorname{Im}(\hat{\omega})$ Approximate \\
\hline 0 & 0.01380 & 0.01380 \\
0.002 & 0.01294 & 0.01294 \\
0.004 & 0.01209 & 0.01209 \\
0.006 & 0.01123 & 0.01123 \\
0.008 & 0.01038 & 0.01038 \\
0.010 & 0.009527 & 0.009523 \\
0.012 & 0.008674 & 0.008668 \\
\hline
\end{tabular}

Table 3: Growth rate of the absolute instability in the $\mathrm{TE}_{12}$ mode in figure 8 as a function of $\delta / r_{w}$. Exact growth rates appear in column 2, and approximate growth rates obtained from (51) appear in column 3. 


\section{FIGURE CAPTIONS}

1. Pulse-shape curves $\left[\operatorname{Im}\left(\hat{\omega}_{s}^{\prime}\right) / \gamma_{v}\right.$ vs. $\left.\beta_{v}\right]$ for the first harmonic of the $T E_{11}$ operating mode. Laboratory-frame values of the parameters appearing in the dispersion relation in (7) are $\beta_{\|}=0.7864, b=0.9102, \epsilon=0.9700 \times 10^{-4}$, and $s=1$. Exact pulse shapes obtained from (15) and (16) are shown by the solid lines. The squares show approximate values obtained from (25) and (26).

2. Temporal growth-rate curve $[\operatorname{Im}(\hat{\omega})$ vs. real $\hat{k}]$ for the first harmonic for the $\mathrm{TE}_{11}$ operating mode. Laboratory-frame values of parameters in the dispersion relation in (7) are the same as those in figure 1.

3. Effect on the $T E_{11}$ pulses in figure 1 of reducing the ratio $\alpha=\beta_{\perp} / \beta_{\|}$while keeping the frequency, applied magnetic field, and the beam energy fixed. The values of $\alpha$ are shown against the curves.

4. Pulse-shape curves $\left[\operatorname{Im}\left(\hat{\omega}_{s}^{\prime}\right) / \gamma_{v}\right.$ vs. $\left.\beta_{v}\right]$ for the first harmonic for the $\mathrm{TE}_{11}$ operating mode when the applied magnetic field is reduced to its grazing value. The frequency, beam current, and kinetic energy are the same as for the case in figure 1 .

5. Temporal growth-rate curves $[\operatorname{Im}(\hat{\omega})$ vs. real $\hat{k}]$ for the first harmonic for the $T E_{11}$ operating mode when the applied magnetic field is reduced to its grazing value. The parameters are the same as those in figure 4.

6. Pulse-shape curves for the first three beam harmonics for the $\mathrm{TE}_{11}$ operating mode. The frequency, applied magnetic field, kinetic energy, and beam current are the same as for the case in figure 1. The quantity $\alpha=\beta_{\perp} / \beta_{\|}=0.33$. The solid squares show approximate pulse shapes for the second harmonic given by (25) and (26).

7. Schematic plot showing the uncoupled beam and waveguide dispersion relations for the $\mathrm{TE}_{13}$ and $T E_{11}$ modes. The $\mathrm{TE}_{13}$ mode is the operating mode. The negative value of $k$ at the lower intersection of the beam and $\mathrm{TE}_{11}$ waveguide mode shows absolute instability in the $\mathrm{TE}_{11}$ mode.

8. Pulse shapes for the $\mathrm{TE}_{13}, \mathrm{TE}_{12}$, and $\mathrm{TE}_{11}$ modes, all normalized to the cutoff frequency of the $\mathrm{TE}_{13}$ mode. The $\mathrm{TE}_{13}$ mode is the operating mode. Absolute instability is present in the $T E_{11}$ and $\mathrm{TE}_{12}$ modes. The solid squares show approximate pulse 
shapes for the $\mathrm{TE}_{11}$ mode given by (25) and (26).

9. Pulse shapes for the $T E_{01}$ and $T E_{11}$ modes, both normalized to the cutoff frequency of the $\mathrm{TE}_{01}$ mode. The $\mathrm{TE}_{01}$ mode is the operating mode. Absolute instability is present in the $\mathrm{TE}_{11}$ mode.

10. Pulse shapes for the $T E_{01}$ mode for various values of $\delta / r_{w}$ shown against the curves. Laboratory frame values of the parameters appearing in (7) are $\beta_{\|}=0.8198, b=$ $0.8591, \epsilon=1.512 \times 10^{-4}$, and $s=1$. A very-slowly-growing absolute instability, present when $\delta / r_{w}=0$, is suppressed by increasing $\delta / r_{w}$ to a value of 0.005 .

11. Plot showing parameter regions of absolute instability for the CRM. The coupling of the beam mode and the waveguide mode produces absolute instability if the point $\left(\beta_{\|}, \epsilon\right)$ lies above the curve of $s b$ for the beam mode harmonic. Two sets of contours of the critical coupling constant are shown; one for $\delta / r_{w}=0$ (solid curves) and one for $\delta / r_{w}=0.01$ (dashed curves).

12. Plot, similar to that in figure 11 , showing exact contours of the critical coupling constant (dashed curves) and approximate contours (solid curves) obtained from (50) for the case of $s b>1$ and $\delta / r_{w}=0.01$.

13. Plot, similar to that in figure 11, showing contours of the critical coupling constant over the full range of $\beta_{\|}$. The curve $A B$ is the locus of points for which $\hat{\omega}_{s}=\hat{k}_{s} / \beta_{\|}$, where $\hat{k}_{s}$ and $\hat{\omega}_{\text {s }}$ are defined in (31) and (32). 


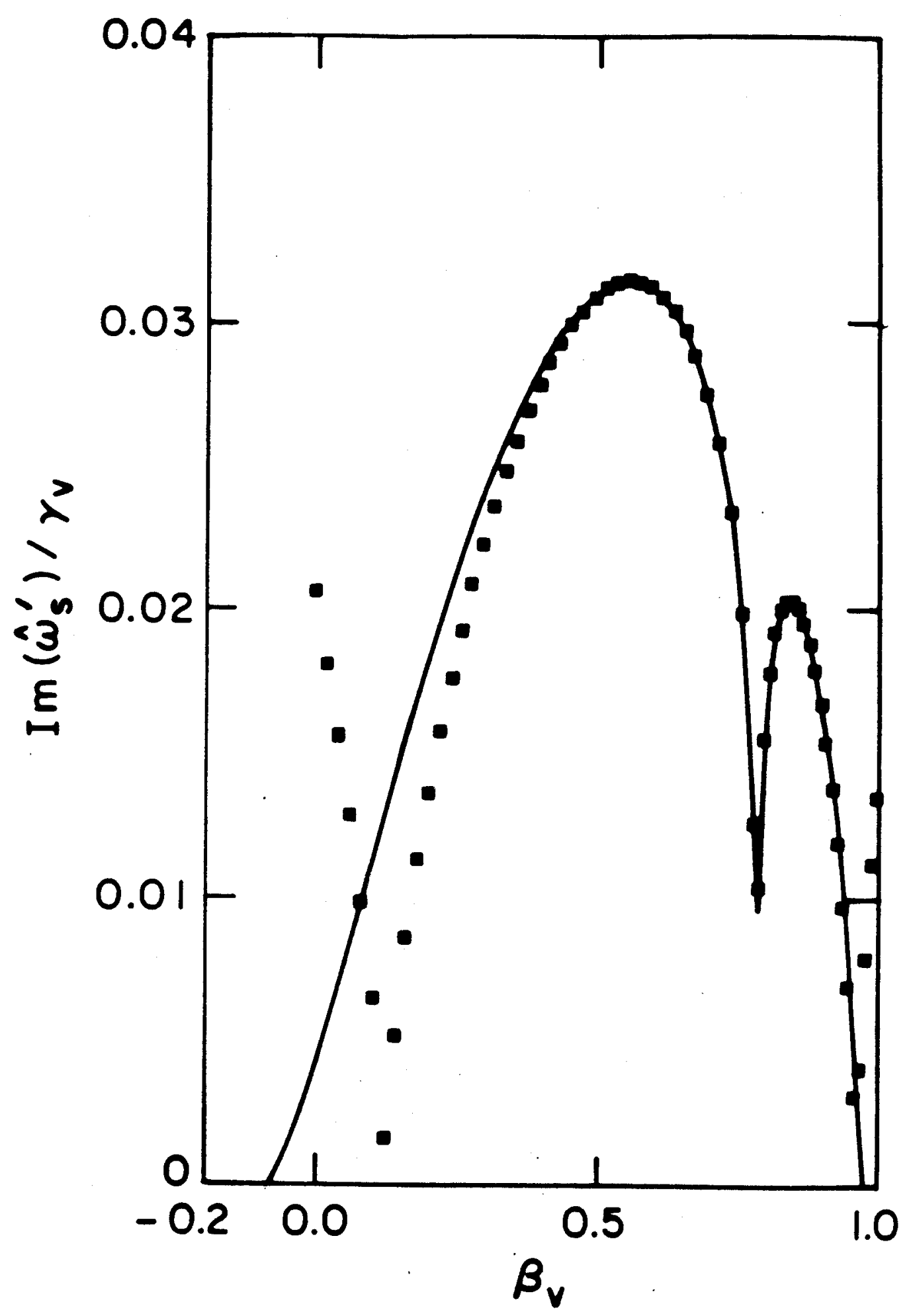

Fig. 1 


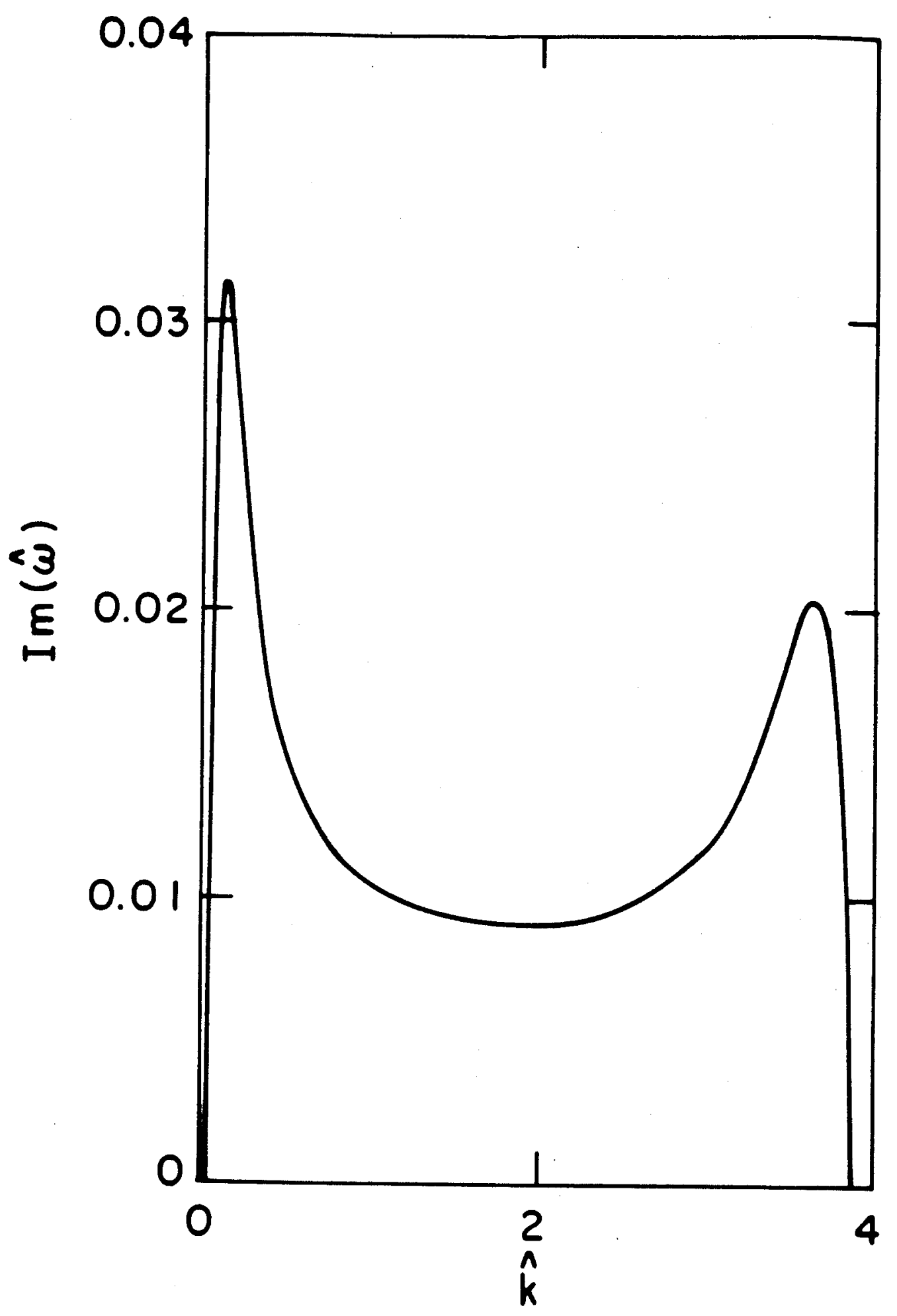

Fig. 2 


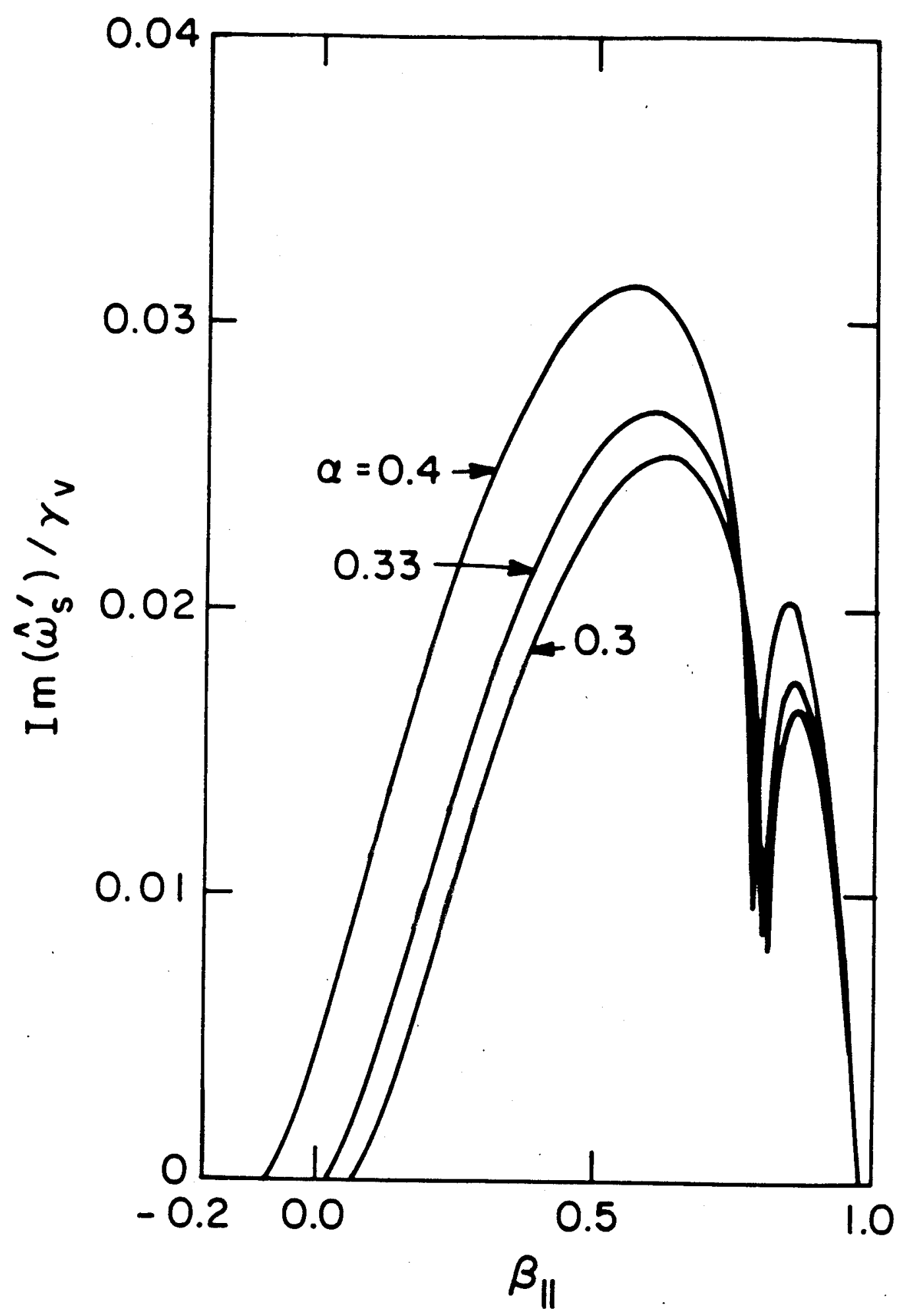

Fig. 3 


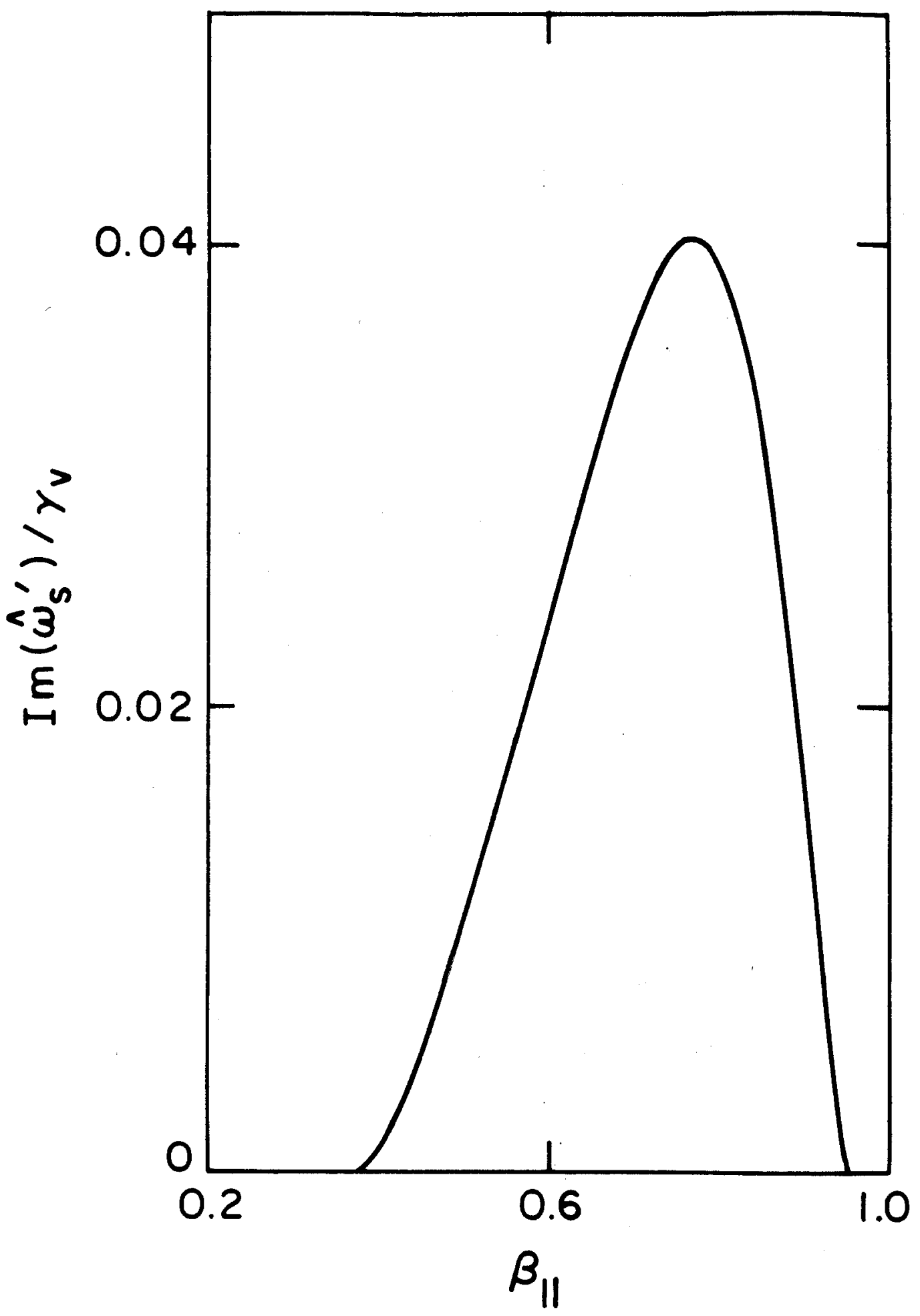

Fig. 4 


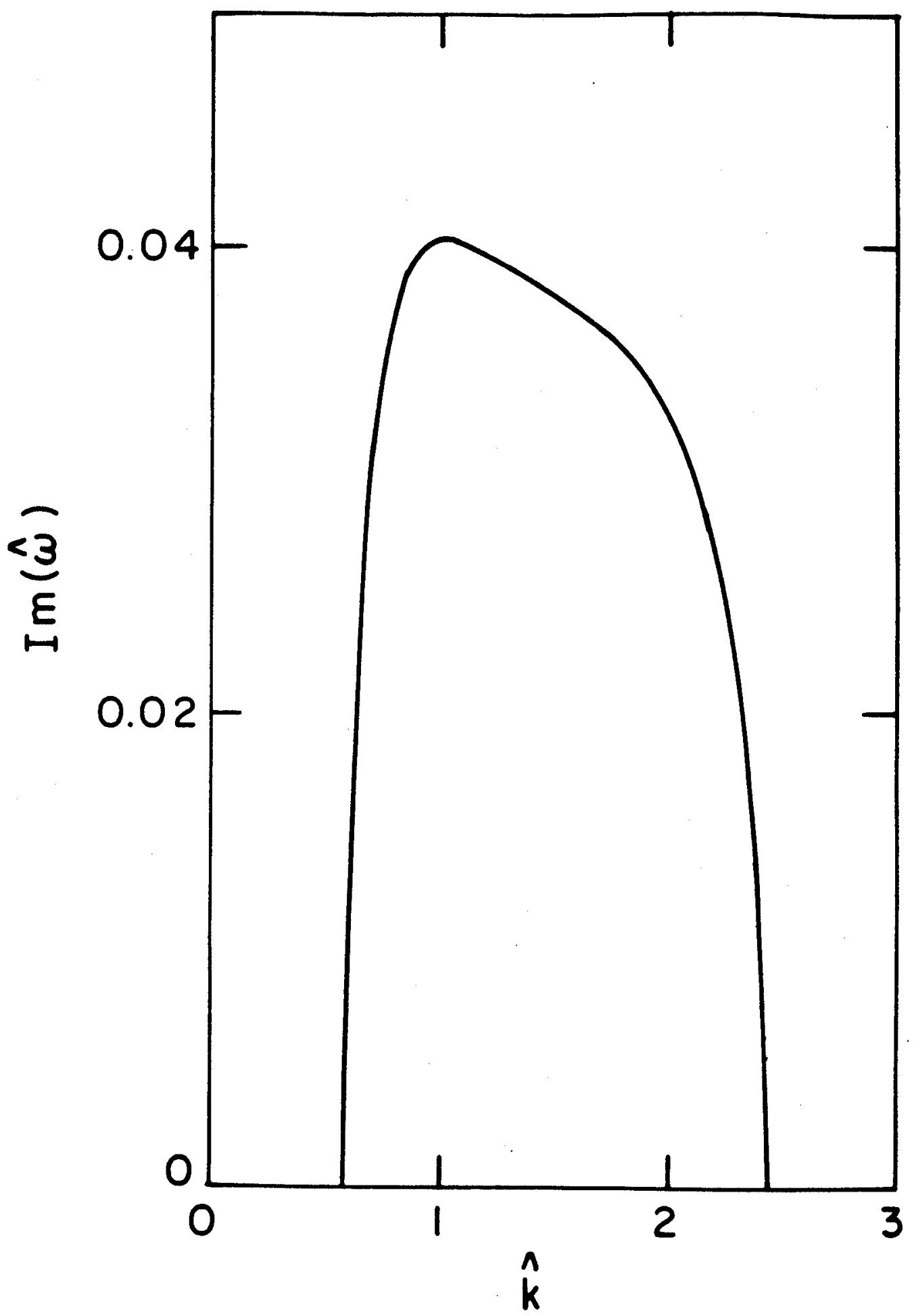

Fig. 5 


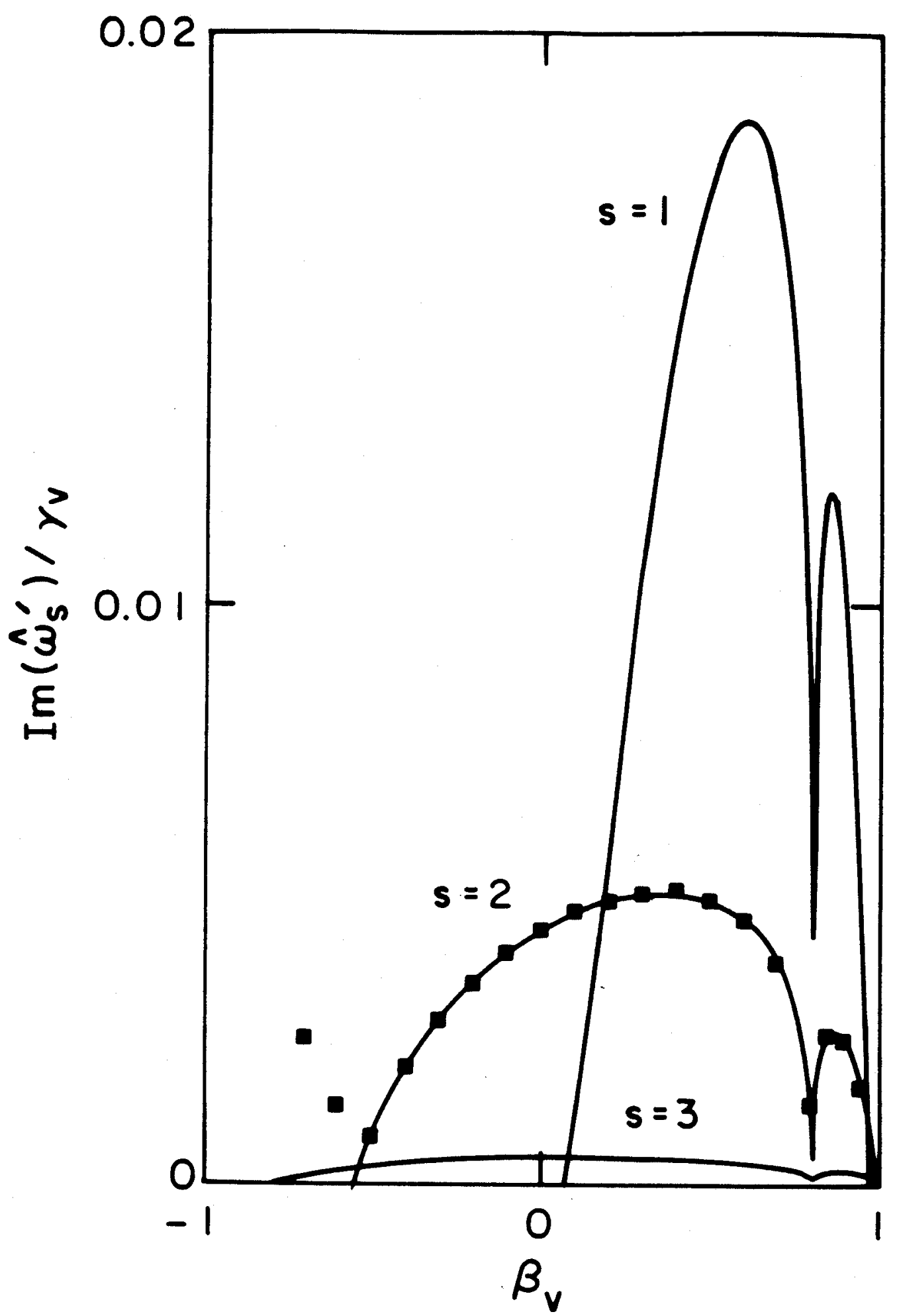

Fig. 6 


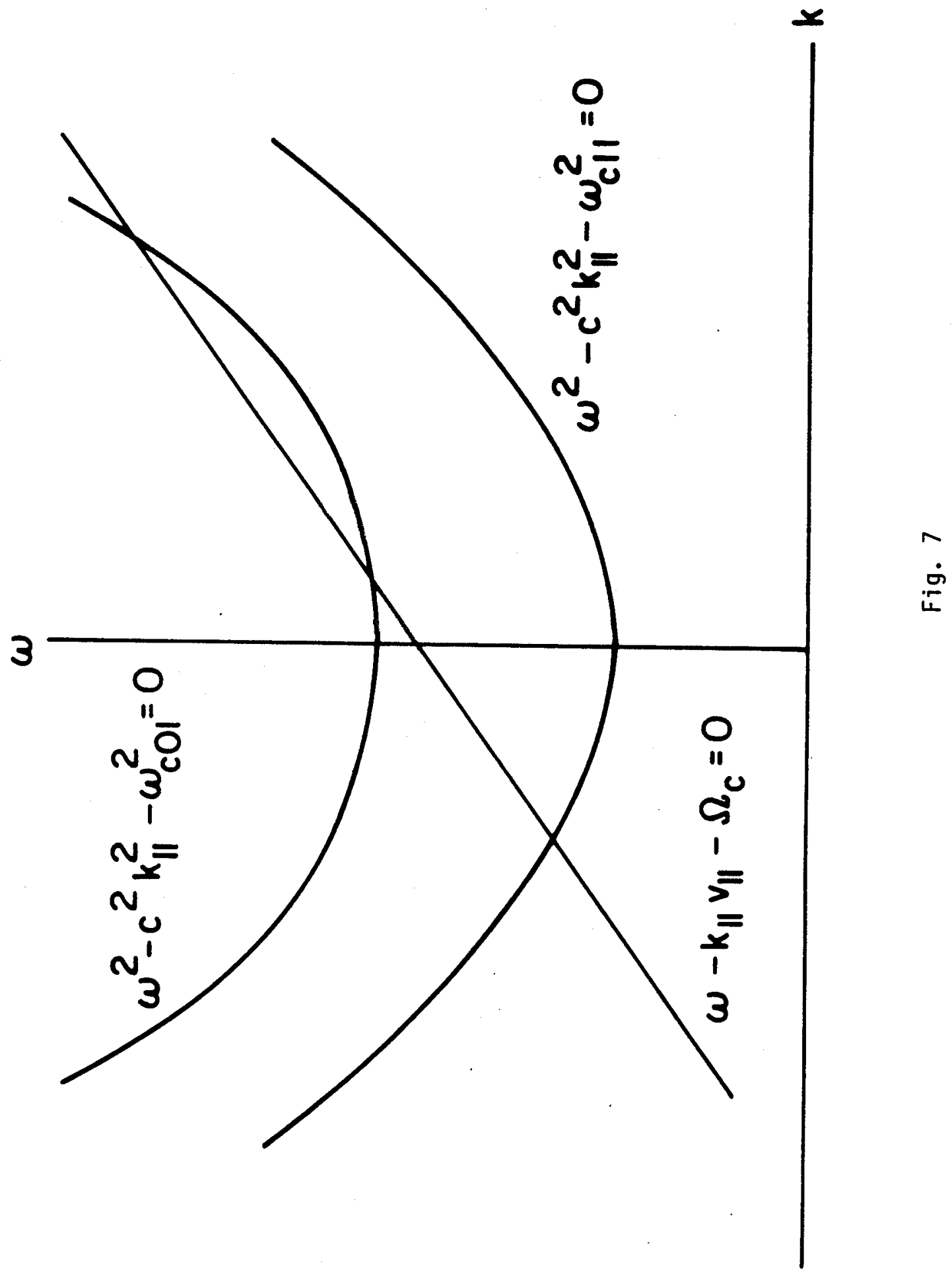




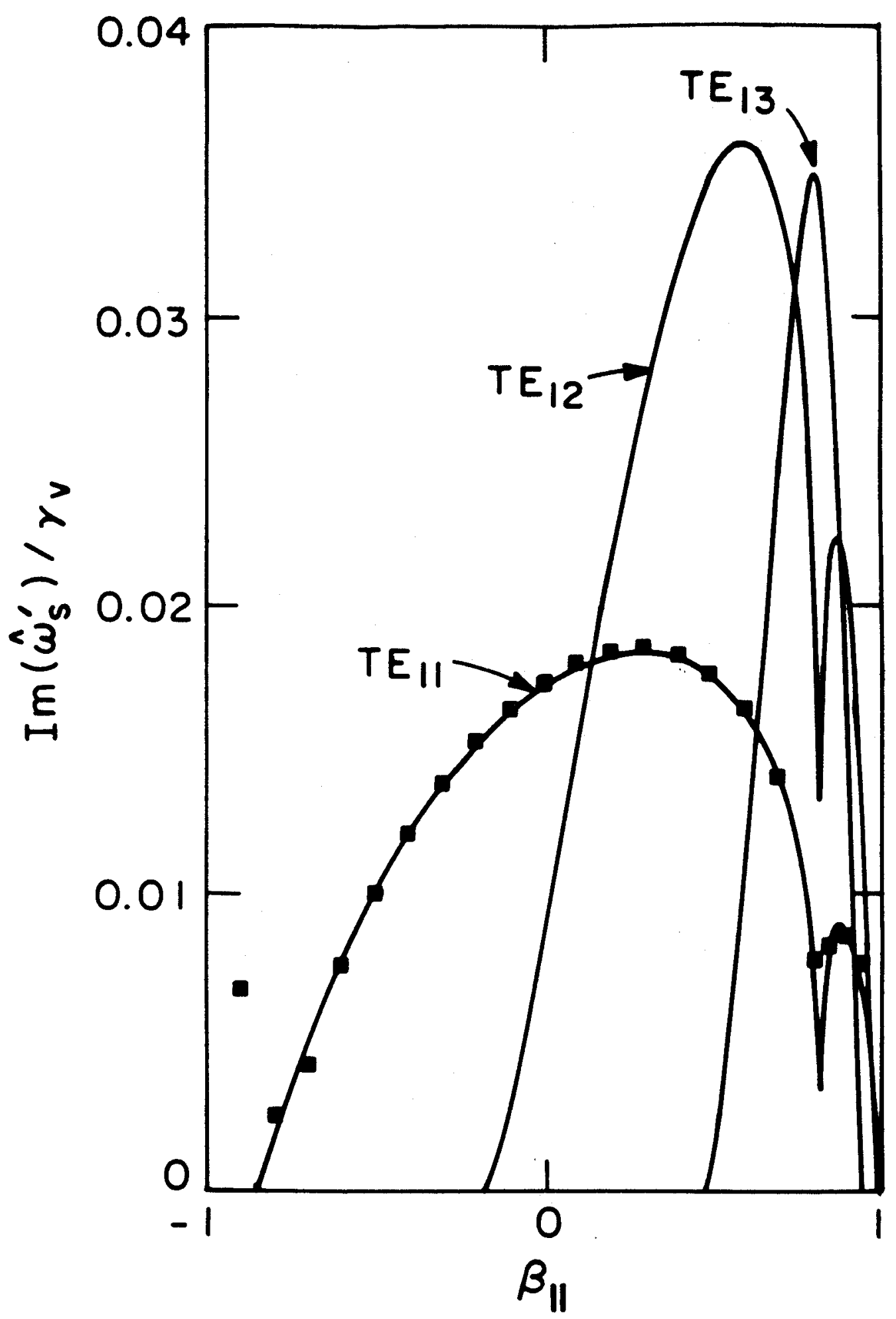

Fig. 8 


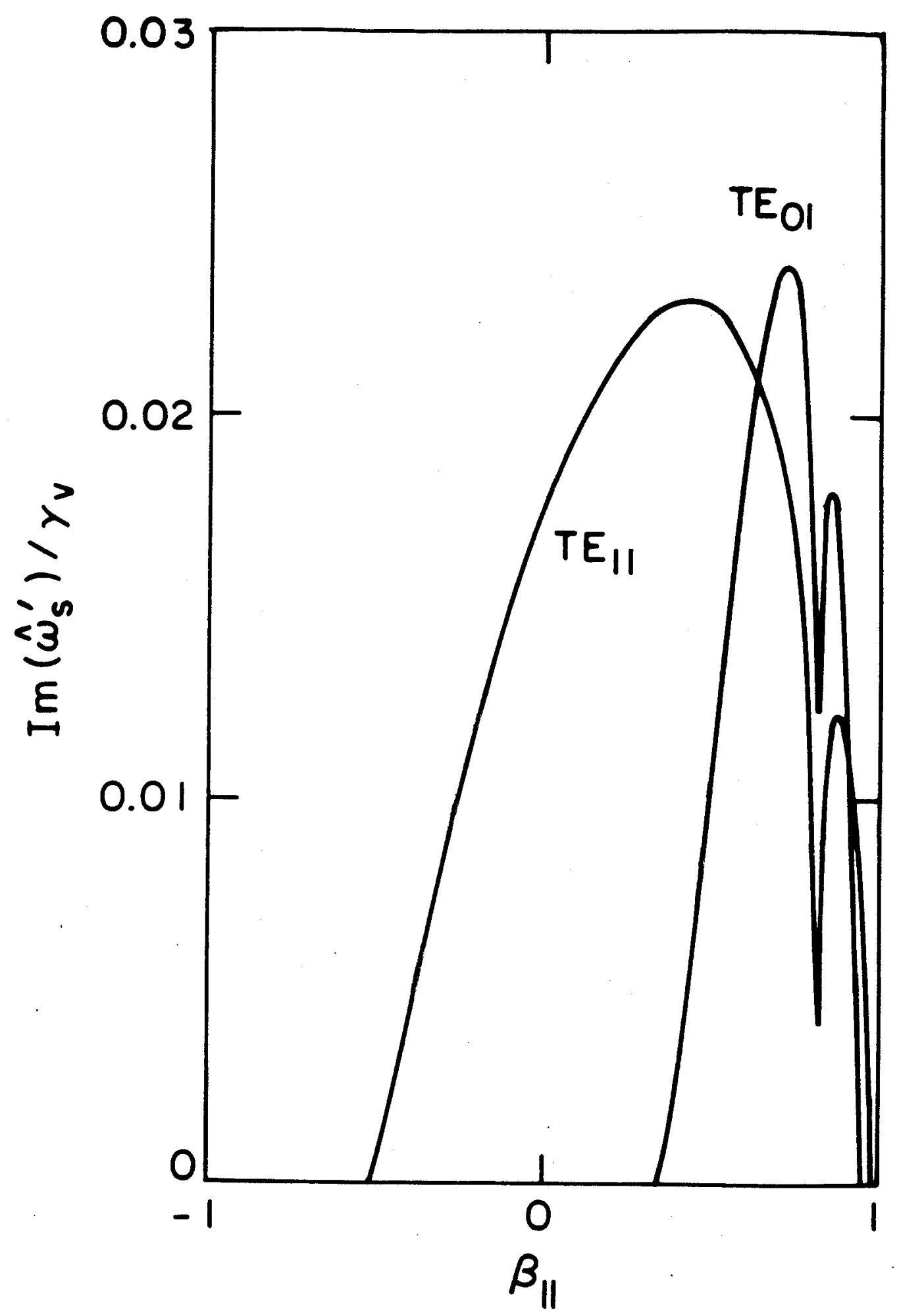

Fig. 9 


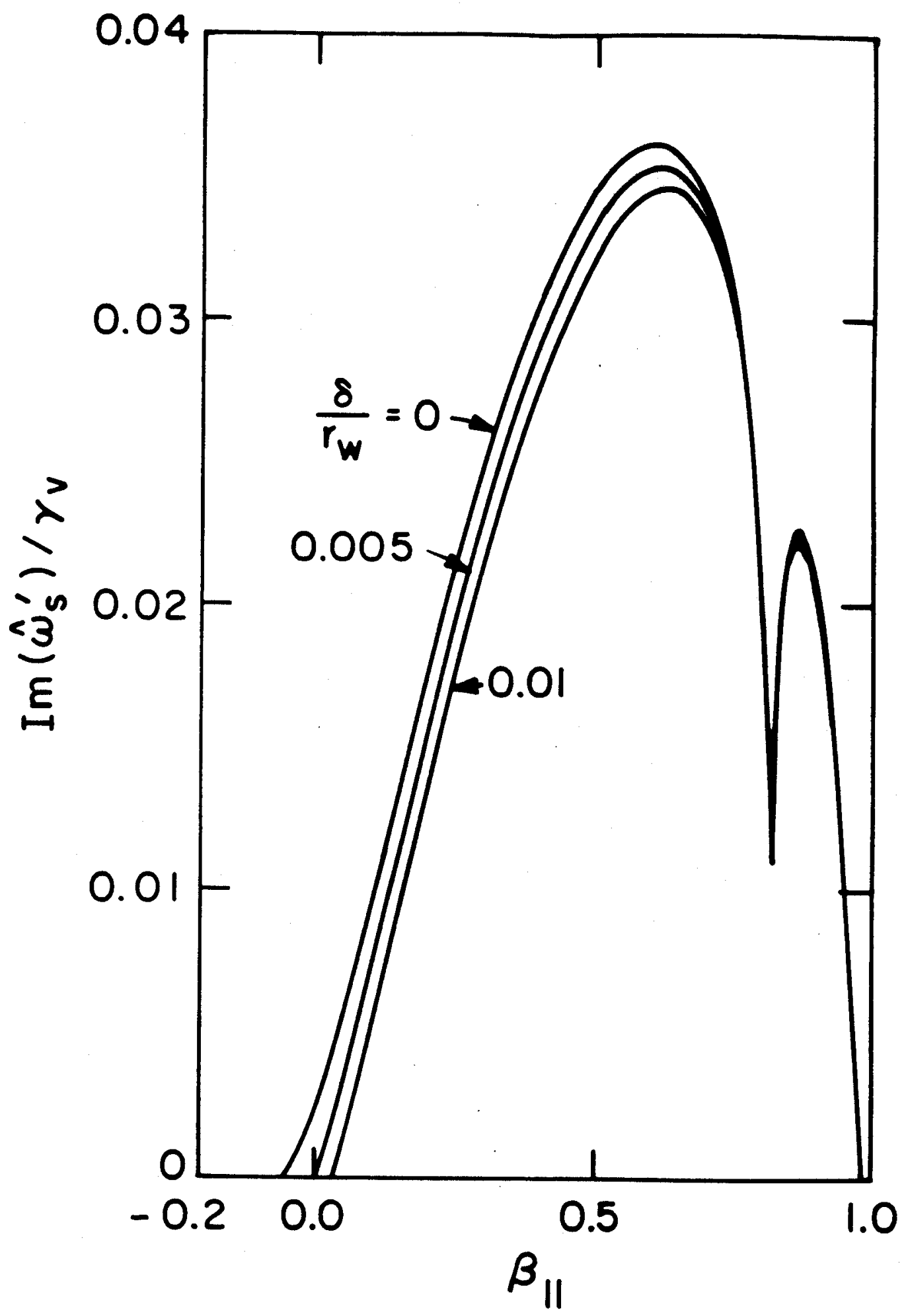

Fig. 10 


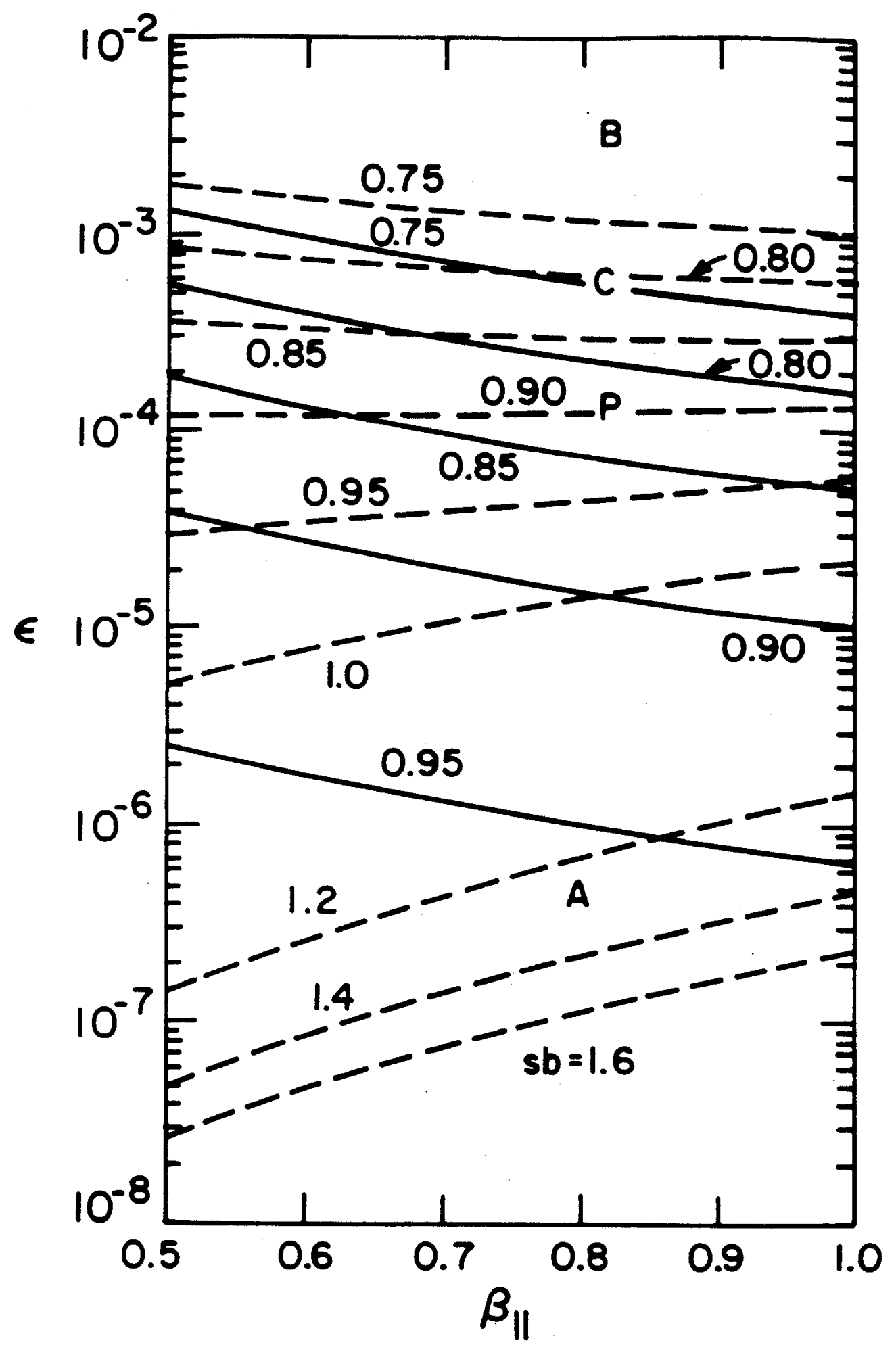

Fig. 11 


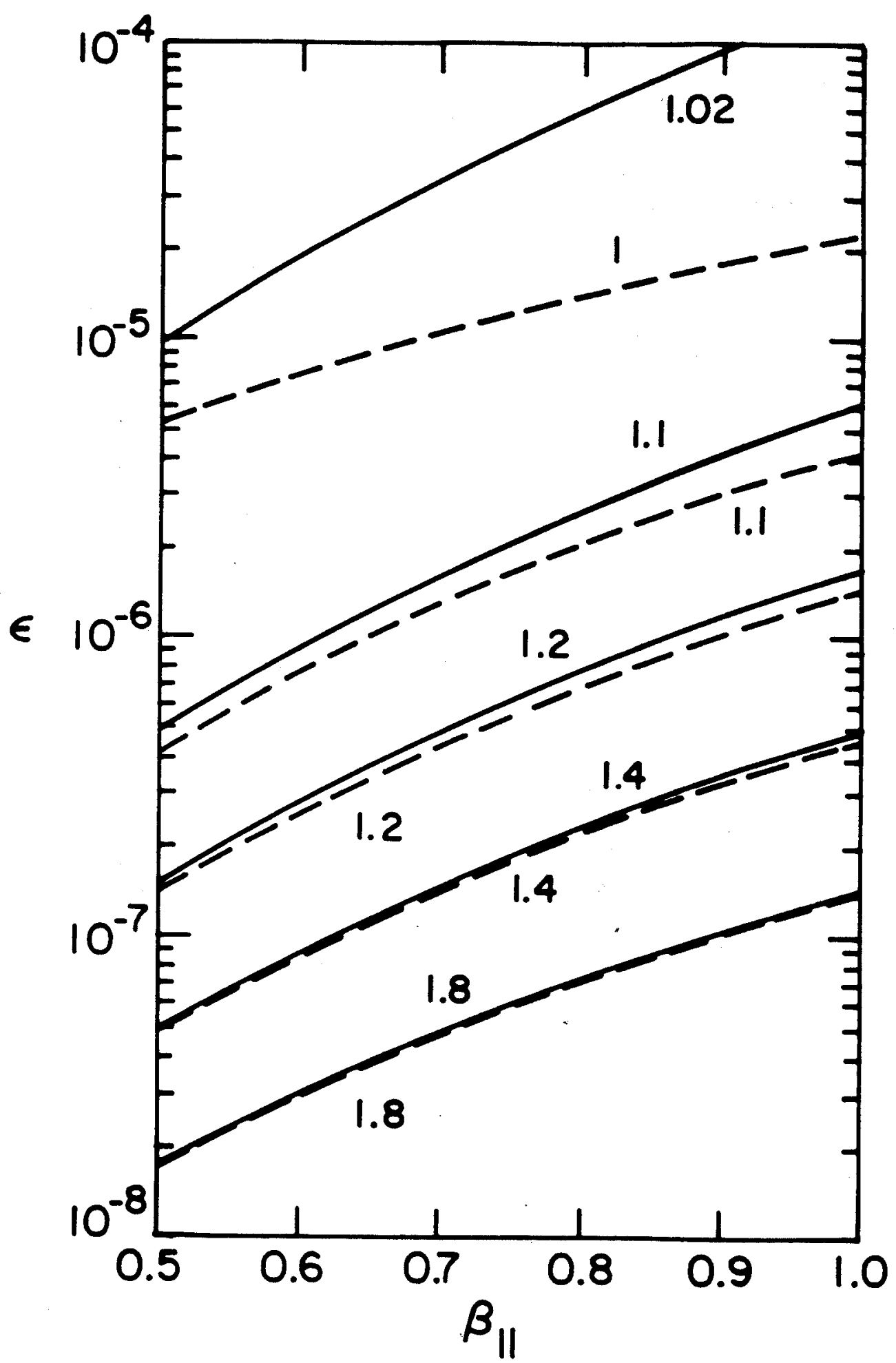

Fig. 12 


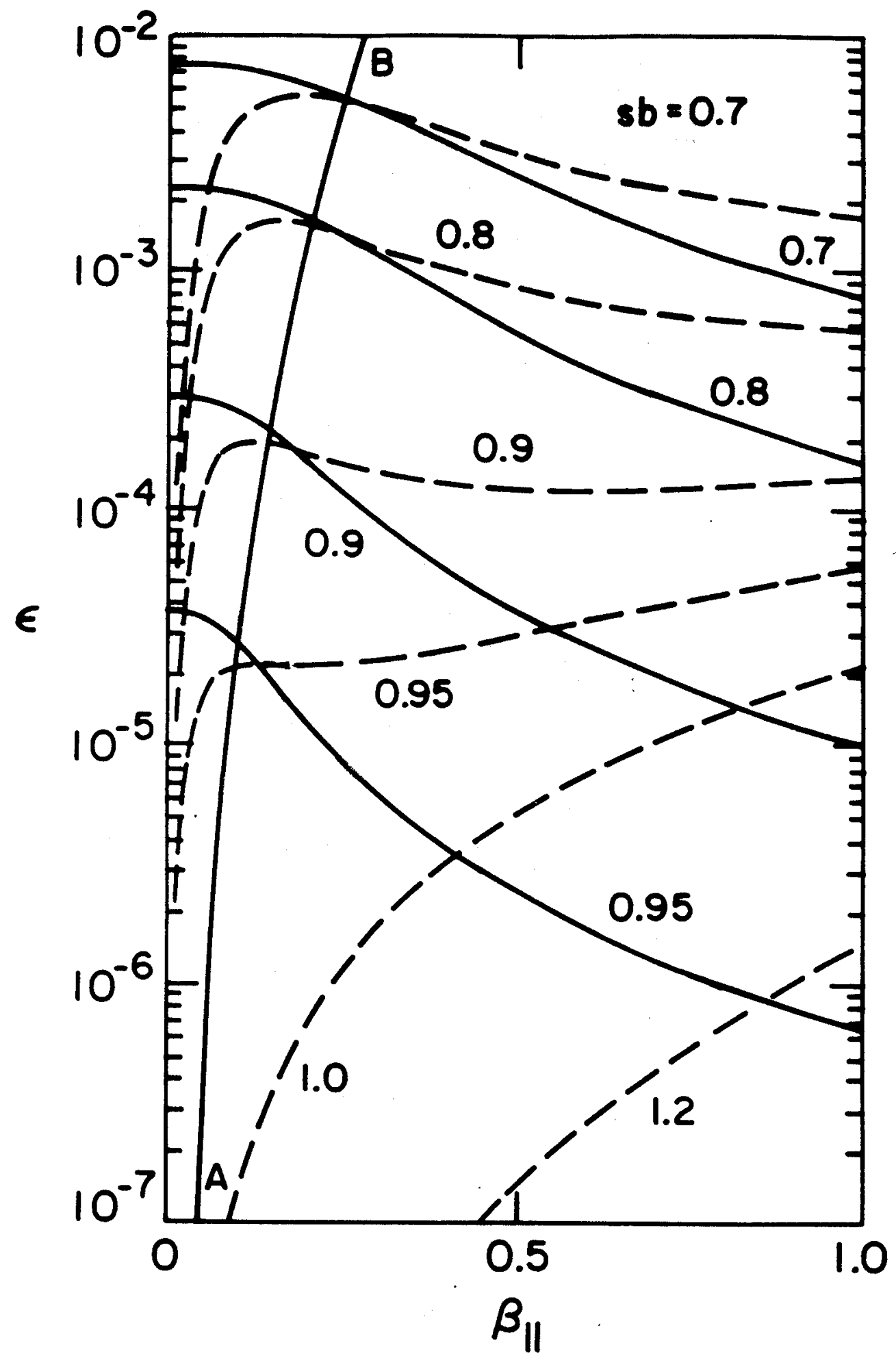

Fig. 13 